\title{
Chromosomal aneuploidies induced upon Lamin B2 depletion are mislocalized in the interphase nucleus
}

\author{
Devika Ranade $^{1} \cdot$ Shivsmriti Koul $^{1} \cdot$ Joyce Thompson $^{1} \cdot$ Kumar Brajesh Prasad $^{1}$. \\ Kundan Sengupta ${ }^{1}$
}

Received: 30 May 2015 / Revised: 28 January 2016 / Accepted: 9 February 2016/Published online: 27 February 2016

(C) The Author(s) 2016. This article is published with open access at Springerlink.com

\begin{abstract}
Chromosome territories assume non-random positions in the interphase nucleus with gene-rich chromosomes localized toward the nuclear interior and gene-poor chromosome territories toward the nuclear periphery. Lamins are intermediate filament proteins of the inner nuclear membrane required for the maintenance of nuclear structure and function. Here, we show using whole-genome expression profiling that Lamin A/C or Lamin B2 depletion in an otherwise diploid colorectal cancer cell line (DLD1) deregulates transcript levels from specific chromosomes. Further, three-dimensional fluorescence in situ hybridization (3D-FISH) analyses of a subset of these transcriptionally deregulated chromosome territories revealed that the diploid chromosome territories in Lamindepleted cells largely maintain conserved positions in the interphase nucleus in a gene-density-dependent manner. In addition, chromosomal aneuploidies were induced in $\sim 25 \%$ of Lamin A/C or Lamin B2-depleted cells. Sub-populations of these aneuploid cells consistently showed a mislocalization of the gene-rich aneuploid chromosome 19 territory toward the nuclear periphery, while gene-poor aneuploid chromosome 18 territory was mislocalized toward the nuclear interior predominantly upon Lamin B2 than Lamin A/C depletion. In addition, a candidate gene locus ZNF570 (Chr.19q13.12) significantly overexpressed upon Lamin B2 depletion was
\end{abstract}

Electronic supplementary material The online version of this article (doi:10.1007/s00412-016-0580-y) contains supplementary material, which is available to authorized users.

Kundan Sengupta

kunsen@ iiserpune.ac.in

1 Biology, Indian Institute of Science Education and Research, Pune, Main Building, Homi Bhabha Road, Pashan,

Pune, Maharashtra 411008, India remarkably repositioned away from the nuclear lamina. Taken together, our studies strongly implicate an overarching role for Lamin B2 in the maintenance of nuclear architecture since loss of Lamin B2 relieves the spatial positional constraints required for maintaining conserved localization of aneuploid chromosome territories in the interphase nucleus.

Keywords Aneuploidy $\cdot$ Chromosome territories $\cdot$ Lamins · Transcription $\cdot$ Cancer cells $\cdot$ Nucleus $\cdot$ Chromosome positioning

\section{Introduction}

Chromosome territories (CTs) assume a unique sub-volume in the three-dimensional interphase nucleus. Gene-rich CTs are positioned toward the nuclear interior, while gene-poor chromosomes are closer to the nuclear periphery (Cremer et al. 2001; Croft et al. 1999). Human CTs 18 ( $>0$ Mbp) and 19 ( $\sim 59 \mathrm{Mbp})$ are of comparable DNA content, but of divergent gene densities of $\sim 8.2$ genes/Mbp and $\sim 37.0$ genes/Mbp, respectively. Chr.18 is peripheral, while Chr.19 is located toward the nuclear center in the interphase nucleus (Cremer et al. 2001; Croft et al. 1999). Such an arrangement is conserved in evolution, suggesting a functional significance for non-random chromosome positions (Neusser et al. 2007; Tanabe et al. 2002). Chromosome positioning is altered in biological processes such as senescence, adipocyte differentiation, spermatogenesis, immune responses, and DNA damage (Foster et al. 2005; Galiova et al. 2004; Mehta et al. 2007, 2013; Szczerbal et al. 2009).

The organization of CTs has been corroborated by highthroughput genomic approaches that map the physical proximity of chromatin contacts as a function of their ligation frequencies (van Berkum et al. 2010). Chromosome 
conformation capture studies ( $3 \mathrm{C}, 4 \mathrm{C}, 5 \mathrm{C}$, and $\mathrm{Hi}-\mathrm{C}$ ) revealed enhanced cis interactions of sub-genomic regions of a chromosome in the nucleus, as opposed to interactions in trans, thereby also suggesting a territorial confinement of chromosomes (Kalhor et al. 2012). Moreover, computational contour maps from chromatin contact frequencies recapitulate a greater enrichment of gene-rich chromosomes toward the nuclear center (Kalhor et al. 2012; Lieberman-Aiden et al. 2009).

Gene loci are significantly smaller $\left(\sim 10^{4}-10^{6}\right.$-fold $)$ than $\mathrm{CTs}$ and exhibit a non-random organization, since overexpressed genes typically "loop-out" of their CT (Chambeyron and Bickmore 2004; Volpi et al. 2000). Hi-C data revealed that the genome is organized in $\sim 1 \mathrm{Mb}$ sized topologically associated domains (TADs) that showed a higher cross-linking frequency with each other as compared to other regions of the genome (Dixon et al. 2012). Gene loci within a TAD have increased proximity in the human X chromosome as shown by three-dimensional fluorescence in situ hybridization (3D-FISH) (Nora et al. 2012), which suggested a concordance between single-cell imaging and 5C. However, the 3D localization of the Hox gene cluster as revealed by 3DFISH showed a greater decompaction as compared to $5 \mathrm{C}$ approaches, suggesting a disagreement between single-cell versus population assays and reiterating the fundamental importance of microscopy-based single-cell assays in a contextspecific manner (Williamson et al. 2014).

The molecular mechanisms that regulate chromosome positioning and genome organization in the interphase nucleus are largely unclear. Lamins, Lamin B receptor (LBR), emerin, actin, and CCCTC-binding Factor (CTCF) have been implicated in the maintenance of chromosome organization and chromatin contacts (Malhas et al. 2007; Meaburn et al. 2007; Ondrej et al. 2008; Phillips-Cremins et al. 2013; Shimi et al. 2008; Solovei et al. 2013; Taimen et al. 2009). Lamins serve to maintain nuclear structure and genome organization. Lamins are type $\mathrm{V}$ intermediate filaments that form coiled coil structures beneath the inner nuclear membrane (Goldman et al. 1986). In higher eukaryotes, A-type Lamins are encoded by a single gene LMNA (that codes for two splice variants - Lamin A and Lamin C) and B type (Lamin B1 and $\mathrm{B} 2$ are encoded by two different genes- $-L M N B 1$ and $L M N B 2$, respectively) (Hoger et al. 1990; Zewe et al. 1991). B-type Lamins are expressed during all stages of development, while A-type Lamins are expressed primarily in differentiated cells (Constantinescu et al. 2006; Rober et al. 1989). Laminopathies are a group of diseases due to mutations in Lamins, which show altered nuclear shapes, gene expression, and chromatin organization (Burke and Stewart 2002; Taimen et al. 2009). Both A- and B-type Lamins are involved in wide range of cellular processes such as replication, transcription, cell division, DNA damage repair, differentiation, and senescence (Butin-Israeli et al. 2015; Martin et al. 2010; Shimi et al. 2011; Shumaker et al. 2008; Spann et al. 2002; Swift et al.
2013; Tang et al. 2008). Furthermore, Lamins exhibit celltype-specific expression levels and function (de Las Heras et al. 2013; Swift et al. 2013; Yang et al. 2011). In addition, the relative stoichiometry of A- and B-type Lamins is often cell-type specific and the ratio of A/B-type Lamins determines mechanical properties of nuclei (Swift et al. 2013).

Nuclear Lamins contribute to the organization of CTs by associating with chromatin in 'Lamina-associated domains' (LADs) during interphase (Guelen et al. 2008). LADs are enriched in Lamina-associated sequences (LASs) and are $\sim 0.1-10-\mathrm{Mb}$ regions characterized by low density of coding genes, high density of repetitive sequences, and inactive histone mark H3K9me2/3 (Belmont et al. 1993; Guelen et al. 2008; Harr et al. 2015; Towbin et al. 2012; Zullo et al. 2012). Constitutive LADs are conserved across cell types and correlate with a repressive state of chromatin (Meuleman et al. 2013). Live imaging assays performed on LADs using a fluorescently coupled m6A-Tracer reveal that they are dynamic, stochastic, and not solely confined to the nuclear periphery, suggesting roles for Lamins in the nuclear interior and periphery (Kind et al. 2013). CTs are mislocalized in cells expressing mutant Lamin A in progeria and cardiomyopathies (Meaburn et al. 2007; Mewborn et al. 2010). Lamin A and B1 depletion in fibroblasts and HeLa cells also showed mislocalized CTs (Malhas et al. 2007; Meaburn et al. 2007; Shimi et al. 2008; Tang et al. 2008).

Furthermore, Lamin A forms a complex with LAP $2 \alpha$ and $\mathrm{BAF}$ and is involved in proper spindle orientation and assembly (Qi et al. 2015). B-type Lamins are also a part of the mitotic spindle matrix in Xenopus laevis and human cells (Goodman et al. 2010; Tsai et al. 2006). Lamin B2 maintains genomic stability and chromosome segregation in colorectal cancer cells (Kuga et al. 2014). Thus, Lamins are unique, since they are required for genome organization, chromosomal stability, and ploidy in mitosis. However, a rather unappreciated role for Lamins is in the spatial organization of diploid and aneuploid chromosome territories in the interphase nucleus.

Aneuploidy is a hallmark of several cancer and developmental disorders. In general, chromosomes assume a genedensity-based positioning pattern in cancer cells (Cremer et al. 2003). More specifically, cancer cells from several epithelial cancers are characterized by aneuploidy with a complex pattern of chromosomal gains and losses (Cimini and Degrassi 2005) that may show changes in chromosome positioning. Notably, CT18 and CT19 are more proximal to one another in colon and cervical cancer cell nuclei as compared to normal cells (Cremer et al. 2003). Chromosomal trisomies generated by artificial introduction of either gene-poor (Chr.7, Chr.18, peripheral) or gene-rich (Chr.19, central) chromosomes assume conserved locations in the nucleus consistent with their gene densities (Sengupta et al. 2007). Human X 
chromosome is altered from a predominantly central to a more peripheral location in X chromosome aneuploidies (XXXXY) (Petrova et al. 2007). Interestingly, while two copies of chromosome 21 territory are in closer proximity as compared to the third copy in cells derived from Down's syndrome patients (Paz et al. 2013), spontaneous trisomy for Chr.12 in human embryonic stem cell line (WA09) also shows an altered position of the trisomic chromosome (Shete et al. 2014). However, given the overarching function of Lamins in regulating ploidy and genome organization, the specific role of Lamins in the spatial organization of aneuploid CTs is largely unclear.

Here, we have studied the role of Lamins in regulation of transcription and spatial organization of the genome in diploid DLD1 cells. Lamin B2 depletion in DLD1 cells shows chromosomal instability (CIN) (Kuga et al. 2014). We show that specific chromosomes are transcriptionally deregulated upon Lamin A/C and Lamin B2 knockdown. Remarkably, transcriptionally deregulated gene-rich or gene-poor chromosomes in Lamin-depleted diploid cells largely assume conserved chromosome positions as revealed by 3D-FISH. However, aneuploid chromosomes were mislocalized in subpopulations of Lamin B2 and not Lamin A/C-depleted cells. In addition, candidate gene loci were repositioned upon Lamin B2 depletion, consistent with an increase in their gene expression levels. Taken together, we propose the involvement of Lamin B2 in mechanisms that regulate spatial organization of aneuploid CTs in the interphase nucleus.

\section{Materials and methods}

\section{Cell culture}

DLD1 colorectal adenocarcinoma cells were obtained from the lab of Thomas Ried, NCI/NIH, Bethesda, USA, and karyotyped independently by 4',6-diamidino-2-phenylindole (DAPI) to ascertain karyotypic stability across passages. DLD1 cells were grown in RPMI 1640 media (Invitrogen, Cat. No. 11875) supplemented with $10 \%$ fetal bovine serum (FBS, Invitrogen, Cat. No. 6140-079 Carlsbad, USA) and antibiotics penicillin $(100 \mathrm{U} / \mathrm{mL})$ and streptomycin $(100 \mu \mathrm{g} /$ $\mathrm{mL}$ ) (Invitrogen, Cat. No. $15070-063$ ) at $37^{\circ} \mathrm{C}$ with $5 \% \mathrm{CO}_{2}$.

\section{Small interfering RNA transfection}

The sequences of the small interfering RNA (siRNA) oligonucleotides targeting Lamins are as follows: $L M N A / C$ : 5'CAGUCUGCUGAGAGGAACA-3', LMNB2: 5'- GAGCA GGAGAUGACGGAGA-3', LMNB 1: 5' - AGACAA AGAGAGAGAGAUG-3' and $L M N B 2$ scrambled: 5'-GG AAGCGUAGACGGAAGAG-3'. DLD1 cells were transfected with $100 \mathrm{nM}$ siRNA oligonucleotide using Lipofectamine RNAiMax (Invitrogen 13778-075) in media with reduced serum (OptiMEM, Invitrogen Cat. No. 31985 070). Control siRNAs used were On-target Plus non-targeting siRNA controls (Dharmacon-D-001810-01-20, D-00181002-20) or siLacZ: 5'-CGUACGCGGAAUACUUCGA-3'; positive control is a siRNA against the gene $P L K 1$ (siPLK1)—polo-like kinase-5'-UGACCUACAUCGA CGAGAA-3'. The uptake of transfection mix was for $6 \mathrm{~h}$, followed by replenishment of complete growth medium. The total duration of the knockdown was for $48 \mathrm{~h}$ at $37^{\circ} \mathrm{C}$.

\section{Western blotting}

Cell lysates were prepared using radioimmunoprecipitation assay (RIPA) buffer and quantified using bicinchoninic acid (BCA) kit (Pierce, Cat. No. 23225). Equal amounts of the protein were boiled in $4 \times$ Laemmli buffer and resolved on a $10 \%$ acrylamide-bisacrylamide gel. The protein was transferred to an activated PVDF membrane at a constant voltage of $90 \mathrm{~V}$ for $90 \mathrm{~min}$. The membrane was blocked in $5 \%$ non-fat dried milk prepared in $1 \times$ Tris-buffered saline-Tween $20(1 \times$ TBST) for $1 \mathrm{~h}$ at room temperature (RT). Primary antibodies used were as follows: Rabbit anti-Lamin A/C (Epitomics (2966-S), 1:5000), Rabbit anti-Lamin B1 (Abcam (ab16048), 1:1000), Mouse anti-Lamin B2 (Abcam (ab8983), 1:400), Mouse anti-Actin (Abcam (ab3280), 1:400), and Rabbit anti-GAPDH (Sigma (G9545), 1:5000). Antibody dilutions were prepared in $0.5 \%$ non-fat dried milk in $1 \times$ TBST and incubated overnight at $4{ }^{\circ} \mathrm{C}$ or for $3 \mathrm{~h}$ at RT. Secondary antibodies used were sheep anti-mouse IgG-horseradish peroxidase (HRP) (GE cat no NA9310V, 1:5000) and donkey anti-rabbit IgG HRP (GE NA9340V, 1:10,000) for $1 \mathrm{~h}$ at RT. Blots were developed using chemiluminescent substrate GE ECL Prime (89168-782), Thermo ECL Western Blot Substrate (32132), and images were acquired at incremental exposures of $10 \mathrm{~s}$ under a chemiluminescence system (LAS4000, GE).

\section{Immunofluorescence assay}

Cells grown on coverslips $\left(18 \times 18\right.$ or $\left.22 \times 22 \mathrm{~mm}^{2}\right)$ were briefly washed using $1 \times$ phosphate-buffered saline $(\mathrm{PBS}, \mathrm{pH}$ 7.4) (5 min, twice at RT) followed by cold cytoskeletal (CSK) buffer $\left(0.1 \mathrm{M} \mathrm{NaCl}, 0.3 \mathrm{M}\right.$ sucrose, $3 \mathrm{mM} \mathrm{MgCl}_{2}, 10 \mathrm{mM}$ PIPES (pH 7.4), $0.5 \%$ Triton X-100) treatment on ice for 5 min. Cells were fixed in $4 \%$ paraformaldehyde (PFA) (prepared in $1 \times$ PBS, pH 7.4) for 10 minutes at RT followed by permeabilization in $0.5 \%$ Triton X-100 (prepared in $1 \times \mathrm{PBS}$ ) for $10 \mathrm{~min}$. Blocking was performed for $30 \mathrm{~min}$ using $1 \%$ bovine serum albumin (BSA) in $1 \times$ PBS at RT. Primary antibodies used were as follows: Rabbit anti-Lamin A/C (Epitomics (2966-S), 1:300), Rabbit anti-Lamin A (Abcam (ab26300), 1:500), Rabbit anti-Lamin B1 (Abcam (ab16048), 1:500), Mouse anti-Lamin B2 (Abcam (ab8983), 
1:600), and Mouse anti-Lamin A (Abcam (ab8980), 1:500). Antibody dilutions were prepared in $0.5 \%$ BSA in $1 \times$ PBS and incubated for $90 \mathrm{~min}$ at RT. Secondary antibodies used were goat anti-rabbit IgG-Alexa 488 (Invitrogen (A-11034) 1:1000) and goat anti-mouse IgG-Alexa 568 (Invitrogen (A11004) $1: 1000$ ) for $1 \mathrm{~h}$ at RT. Cells were counter stained with DAPI for 2 min at RT, washed in $1 \times$ PBS, mounted in Slowfade Gold Antifade (Invitrogen S36937), and stored in $4{ }^{\circ} \mathrm{C}$ until they were imaged. Quantification of fluorescence intensities of the acquired images was performed using ImageJ with line scans manually drawn across nuclei.

\section{Three-dimensional fluorescence in situ hybridization-chromosome territories}

\section{Fixation and permeabilization}

Cells were grown to a confluency of $\sim 30-40 \%$ on glass coverslips $\left(18 \times 18\right.$ or $\left.22 \times 22 \mathrm{~mm}^{2}\right)$ placed in single wells of a sixwell plate and were subjected to siRNA knockdown (Kd). Cells were washed three times in $1 \times$ PBS for 5 min each at RT. The cells were incubated on ice for $5 \mathrm{~min}$ in pre-chilled CSK buffer and immediately fixed in $4 \%$ paraformaldehyde (PFA, prepared in $1 \times \mathrm{PBS}(\mathrm{pH} 7.4)$ ) for $7 \mathrm{~min}$ at RT. The cells were washed in $0.1 \mathrm{M}$ Tris- $\mathrm{HCl}(\mathrm{pH}$ 7.4) followed by two washes with $1 \times$ PBS for 5 min each at RT. The cells were repermeabilized in $0.5 \%$ Triton X-100 (prepared in $1 \times \mathrm{PBS}$ ) for $10 \mathrm{~min}$ and incubated in $20 \%$ glycerol (prepared in $1 \times$ PBS) for 60 min followed by four freeze-thaw cycles in liquid nitrogen. The cells were washed three times in $1 \times$ PBS for 5 min each and incubated in $0.1 \mathrm{~N} \mathrm{HCl}$ for $10 \mathrm{~min}$ followed by three washes in $1 \times$ PBS for 5 min each. The cells were stored in $50 \%$ formamide $(\mathrm{FA}) / 2 \times$ saline sodium citrate $(\mathrm{SSC})(\mathrm{pH}$ 7.4) overnight at $4{ }^{\circ} \mathrm{C}$ or until used for hybridization.

\section{Hybridization}

Chromosome painting probes were obtained from Applied Spectral Imaging (ASI), Israel, or MetaSystems, USA. Probes were equilibrated at $37^{\circ} \mathrm{C}$ for $5 \mathrm{~min}$ followed by denaturation at $80^{\circ} \mathrm{C}$ for $5 \mathrm{~min}$ and quick chilled on ice for $2 \mathrm{~min}$ followed by a pre-annealing at $37^{\circ} \mathrm{C}$ for $30 \mathrm{~min}$. This denatured probe $(3-4 \mu \mathrm{L})$ was spotted onto fixed cells, sealed, and subjected to co-denaturation at $80^{\circ} \mathrm{C}$ for $5 \mathrm{~min}$. Hybridization was for $48 \mathrm{~h}$ in a humidified box at $37^{\circ} \mathrm{C}$.

\section{Detection}

Post hybridization, coverslips were washed in $50 \% \mathrm{FA} / 2 \times$ SSC (pH 7.4), thrice for $5 \mathrm{~min}$ each at $45^{\circ} \mathrm{C}$, followed by three washes for $5 \mathrm{~min}$ each in $0.1 \times \mathrm{SSC}$ at $60^{\circ} \mathrm{C}$ with gentle agitation. Coverslips were briefly rinsed in $0.1 \%$ Tween 20/ $4 \times \mathrm{SSC}$ and counterstained with DAPI for 2 min, washed in
$2 \times \mathrm{SSC}$ and mounted in Slowfade Gold Antifade (Invitrogen S36937), and stored at $4{ }^{\circ} \mathrm{C}$ until they were imaged.

\section{Imaging}

Image acquisition was performed on a Zeiss LSM 710 or 780 confocal microscope (Carl Zeiss, Thornwood, NJ, USA) with $\mathrm{a} \times 63$ Plan-Apochromat 1.4 NA oil immersion objective using scan zoom of 2.5. Acquisition of Z-stacked images (voxel size of $0.105 \mu \mathrm{m} \times 0.105 \mu \mathrm{m} \times 0.34 \mu \mathrm{m}$ ) was at $512 \times 512$ pixels per frame using 8 -bit pixel depth for each channel. The line averaging was set to 4 , and images were collected sequentially in a three-channel mode.

\section{Three-dimensional immunofluorescence in situ hybridization-gene loci}

\section{Preparation of bacterial artificial chromosome DNA probes}

Bacterial artificial chromosome (BAC) DNA was extracted from clones purchased from CHORI BACPAC Resources (using Hi-Pure Plasmid DNA Extraction Kit (Invitrogen K210017). BAC clones used were RP11-1134K12 for ZNF570 gene. This DNA was nick translated using fluorescently labeled dUTP (Abbott) and Nick Translation Mix (Roche 11745808 910). Labeling reaction was at $15^{\circ} \mathrm{C}$ for $90 \mathrm{~min}$, followed by termination of reaction using $0.5 \mathrm{M}$ EDTA (pH 8.0) at $65^{\circ} \mathrm{C}$ for $10 \mathrm{~min}$. The DNA was precipitated using ethanol and $3 \mathrm{M}$ sodium acetate and resuspended using deionized FA and Master Mix containing dextran sulfate and $2 \times$ saline sodium citrate (SSC) buffer.

\section{Fixation and permeabilization of cells}

Cells grown on coverslips and siRNA treated for $48 \mathrm{~h}$ were washed twice ( 5 min each) with $1 \times$ PBS ( $\mathrm{pH} 7.4$ ) followed by permeabilization on ice using ice-cold CSK buffer containing $0.5 \%$ Triton X-100 for 5 min. Cells were subsequently fixed in $4 \%$ paraformaldehyde (PFA) solution prepared in $1 \times \mathrm{PBS}$ for 5-7 min at RT. The fixation protocol followed was the same as that for 3D-FISH (described above in 3D-FISH for visualizing $\mathrm{CTs}$ ).

\section{Immunostaining followed by hybridization}

Previously fixed coverslips were subjected to two washes with $1 \times$ PBS (5 min each). Blocking was performed for 30 min using $1 \%$ bovine serum albumin (BSA) in $1 \times$ PBS at RT. Primary antibodies used were as follows: Mouse anti-Lamin B2 (Abcam (ab8983), 1:600) and Rabbit anti-Lamin A (Abcam (ab26300), 1:500). Antibody dilutions were prepared in $0.5 \%$ BSA in $1 \times$ PBS and incubated for $90 \mathrm{~min}$ at RT. Secondary 
antibodies used were goat anti-mouse IgG-Alexa 633 (Invitrogen (A-21052) 1:1000) and goat anti-rabbit IgGAlexa 488 (Invitrogen (A-11034) 1:1000) for $1 \mathrm{~h}$ at RT. Post fixation and post permeabilization were subsequently performed with $4 \%$ PFA (5 min RT) and $0.5 \%$ Triton X100 in $1 \times$ PBS (5 min RT), respectively. This was followed by two washes each with $1 \times$ PBS (5 min each) and $50 \% \mathrm{FA} / 2 \times \mathrm{SSC}(\mathrm{pH} 7.4)$. The probe for ZNF570 was equilibrated at $37{ }^{\circ} \mathrm{C}$ for 5 min followed by denaturation at $80^{\circ} \mathrm{C}$ for $5 \mathrm{~min}$ and quick chilled on ice for $2 \mathrm{~min}$. Preannealing was performed at $37{ }^{\circ} \mathrm{C}$ for $30-40 \mathrm{~min}$. This denatured probe $(3-4 \mu \mathrm{L})$ was spotted onto the fixed cells that were subjected to immunostaining for Lamin A and Lamin B2 and subjected to co-denaturation at $80^{\circ} \mathrm{C}$ for $5 \mathrm{~min}$. Hybridization was for $48 \mathrm{~h}$ in a humidified box at $37{ }^{\circ} \mathrm{C}$.

\section{Post hybridization washes}

Post hybridization, coverslips were washed in $50 \% \mathrm{FA} / 2 \times$ SSC (pH 7.4), thrice for 5 min each at $45{ }^{\circ} \mathrm{C}$, followed by three washes for $5 \mathrm{~min}$ each in $0.1 \times \mathrm{SSC}$ at $60^{\circ} \mathrm{C}$ with gentle agitation. Coverslips were stained with DAPI and mounted with Antifade and stored in $4{ }^{\circ} \mathrm{C}$ until they were imaged. The images were acquired on Zeiss LSM 710 or 780 confocal microscope similarly as for CT hybridizations, using a zoom $=2.0$.

\section{Radial distance measurements of chromosome territories}

Three-dimensional distance measurements of CTs were performed using Image-Pro Plus (v 7.1), Media Cybernetics, USA. Briefly, LSM files containing optical sections $(z=0.34 \mu \mathrm{m})$ of the hybridized nuclei were subjected to 3D surface rendering. Three-dimensional reconstructions of each nucleus were performed on individually cropped nuclei. The acquired images were thresholded and surface rendered for each of the red, green, and blue channels. The geometric center of the DAPI-stained nucleus (blue channel) and the CTs (red and green channels) were determined using plugins from the software, and the distance between the geometric center of the nucleus (A) and that of the territory (B) was measured (X). The vector from the geometric center of nucleus (A) to the geometric center of the CT (B) was extended to a third collinear point at the nuclear periphery $(\mathrm{C})$. The distance between the geometric center (A) of the nucleus and $(\mathrm{C})$ was calculated $(\mathrm{Y})$. The relative distance of a CT from the center of the nucleus was calculated as a percentage of the total distance from the center of the nucleus to the nuclear periphery $(\mathrm{Y})$, \% radial distance $(\mathrm{RD})=(X / Y) * 100$ (Tanabe et al. 2002).

\section{Measurements for spatial organization of gene loci}

\section{Distance measurement from the nuclear periphery}

Distances of gene loci from the nuclear periphery were measured (in $\mu \mathrm{m}$ ) in 3D using Lamin A signal at the nuclear periphery to demarcate the edge of the nucleus. Briefly, surface rendering was performed for the gene locus (red channel), Lamin A (green channel), Lamin B2 (far-red channel), and the nucleus (DAPI signal blue channel) using Huygens Professional software. For Lamin B2 knockdown (Kd) cells, the nuclei which showed a depletion of Lamin B2 (far-red channel) were used for analysis. Briefly, surface rendering for Lamin A was used as an anchor for measurements. Center of mass $(\mathrm{CM})$ was determined for the gene locus signal of interest, and the closest distance between the $\mathrm{CM}$ and surface of the anchor (Lamin A signal) was measured.

\section{Statistical analysis}

Graphs were plotted using Graph Pad Prism 5.0 and Sigma Plot 12.0. Statistical comparisons were performed using Graph Pad Prism 5.0 software. Fisher's exact and $\chi^{2}$ test was used to compare the distribution of \% radial distances (RD) of CTs binned in five nuclear sub-shells. For comparison of volumes of CTs and the nucleus between diploid and aneuploid subpopulations, non-parametric ANOVA Kruskal-Wallis test was used. The distance of gene locus ZNF570 from the lamina was compared using two-sample Kolmogorov-Smirnov (KS) test between control and Lamin B2-depleted cells; $p$ value $<0.05$ was considered to be statistically significant.

\section{RNA extraction and quantitative reverse transcription-PCR}

RNA extraction was performed using PureLink RNA Mini Kit (12183018A). cDNA was synthesized using ImProm II Reverse Transcriptase system (Promega A3800). Quantitative real-time PCR was performed using SYBR Green (SAF Labs). Sequences of primers are in Table S1. $A C T I N$ and GAPDH served as internal controls.

\section{Gene expression profiling using microarrays}

Briefly, RNA was extracted from three independent biological replicates each from control (untreated), Lamin $\mathrm{A} / \mathrm{C} \mathrm{Kd}$, and Lamin B2 Kd DLD1 cells, and its quality was ascertained using bioanalyzer (Agilent). Cy3-labeled complementary RNA was prepared using T7 promoter-based linear amplification (Agilent Quick Amp Labeling Kit, Cat No. 5190-0442). Qiagen RNeasy Mini Kit (Cat No. 74104) was used for RNA purification. Hybridization was performed using a human $8 \times$ $60 \mathrm{~K}$ array (Agilent single-color 27114) and labeled using 
Agilent's in situ hybridization kit (Cat No. 5188-5242). Array scanning was performed, and data acquired was normalized (50th percentile shift normalization). Analyses of gene expression levels were based on comparison of hybridizations between knockdown and control (untreated DLD1 cells) as reference. Gene expression levels (absolute fold change $\geq 2.0$, $p<0.05$ ) were used for further analyses. The microarray data can be accessed using GEO Accession number GSE73269. The $\%$ deregulation per chromosome was calculated as (no. of deregulated genes on a chromosome/total no. of coding genes on that chromosome) $\times 100$. Number of coding genes and size of chromosomes were obtained from MapViewer (NCBI). Chromosomes showing $>0.7 \%$ deregulation were considered significant since this represents $>50 \%$ of the total extent of transcriptional deregulation.

\section{Preparation of metaphase spreads}

Cells (control, Lamin Kd) were blocked in metaphase using Colcemid $(0.1 \mu \mathrm{g} / \mathrm{mL})$ for $90 \mathrm{~min}$. Hypotonic treatment $(0.075 \mathrm{M} \mathrm{KCl})$ was performed for $30 \mathrm{~min}$ at $\mathrm{RT}$, followed by fixation in five to six drops of fixative (methanol/acetic acid 3:1), followed by three washes in fixative. Cells were dropped onto glass slides, and metaphases were stained with DAPI, imaged, and counted.

\section{Fluorescence-activated cell sorting analysis by propidium iodide staining}

Cells (control, Lamin Kd) were fixed in $70 \%$ ethanol (in $1 \times$ PBS), subjected to RNase treatment along with propidium iodide staining $(1 \mathrm{~h})$ on ice. Cell suspensions were subsequently run on FACSCalibur (BD Biosciences).

\section{RNA fluorescence in situ hybridization}

Probe preparation The probe for RNA FISH for ZNF570 was prepared by nick translation of the BAC clone ("Preparation of bacterial artificial chromosome DNA probes" section-3D FISH for gene loci above). Upon precipitation, the DNA was resuspended in $8 \mu \mathrm{L}$ of deionized FA and stored at $-20{ }^{\circ} \mathrm{C}$ till further use. For hybridization, the probe was equilibrated at $37^{\circ} \mathrm{C}$ for $5 \mathrm{~min}$ followed by denaturation at $80{ }^{\circ} \mathrm{C}$ for $5 \mathrm{~min}$, mixed with equal volume of $2 \times$ hybridization mix containing vanadyl ribonucleoside complex, and incubated on ice for $30 \mathrm{~min}$.

Fixation and hybridization Cells were washed twice in $1 \times$ PBS ( 5 min each), followed by treatment with CSK buffer on ice for $5 \mathrm{~min}$ and fixation using $4 \%$ PFA ( 7 min RT). The cells were washed with $70 \%$ ethanol twice and stored at $-20{ }^{\circ} \mathrm{C}$ until further use. Prior to hybridization, the cells were subjected to an ethanol series (70-90-100\% ethanol) and air-dried.
The probe was added to cells and incubated at $37^{\circ} \mathrm{C}$ overnight, followed by washes with $50 \% \mathrm{FA} / 2 \times \mathrm{SSC}$ and $2 \times \mathrm{SSC}(\mathrm{pH} 7.2$ 7.4) at $42{ }^{\circ} \mathrm{C}$ (three washes each of $5 \mathrm{~min}$ each). The cells were mounted using DAPI-Antifade. All reagents for RNA FISH were prepared using DEPC-treated water (Chaumeil et al. 2008).

\section{Results}

\section{Specific chromosomes are transcriptionally deregulated upon Lamin A/C and Lamin B2 depletion}

Lamins are filamentous proteins that localize beneath the inner nuclear membrane and maintain nuclear structure and function (Adam and Goldman 2012). In addition to the nuclear periphery, Lamins exist in the nucleoplasm as a meshwork that extends into the nuclear interior and associate directly or indirectly with LAP2 $\alpha$, BANF1, PCNA, and transcription factors (Dechat et al. 2000; Lee et al. 2001; Shumaker et al. 2008). The role of Lamins is therefore not just confined to the nuclear periphery but significantly extends to the nuclear interior, owing to its interaction with proteins at both these nuclear locations (Gruenbaum and Medalia 2015; Kolb et al. 2011). A- and B-type Lamins are implicated in DNA replication and repair, transcription, and senescence (Moir et al. 1994; Shimi et al. 2011; Shumaker et al. 2008; Tang et al. 2008).

We sought to examine the impact of Lamin depletion on the transcriptome and spatial organization of chromosomes in otherwise diploid colorectal cancer cells (DLD1), which are karyotypically stable across passages, distinguishing the same from other cell lines with severe chromosomal aberrations. We first established the conditions for Lamin depletion in DLD1 cells by performing siRNA-mediated knockdowns (Kd). Lamin Kd showed a viability of $\sim 60-70 \%$ in DLD1cells (Fig. S1a). A knockdown of $>80-90 \%$ was ascertained at the transcript level by quantitative reverse transcription (qRT)-PCR (Fig. 1a), protein level by immunoblotting (Figs. $1 \mathrm{~b}$ and $\mathrm{S} 1 \mathrm{~b}-\mathrm{d}$ ), and in single cells by immunofluorescence staining (Fig. 1c-f). Immunoblots revealed that Lamin $\mathrm{A} / \mathrm{C} \mathrm{Kd}$ did not significantly affect the levels of B-type Lamins (Figs. 1b and S1b). Likewise, depletion of Lamin B1 or B2 did not affect Lamin A/C levels (Fig. 1b). The exclusivity of the knockdowns enabled us to interpret the consequences of a single Lamin depletion. This is noteworthy since expression levels and stoichiometry of Lamins are important determinants of Lamin localization and function in a cell-type-dependent manner (Guo et al. 2014; Shimi et al. 2015; Swift et al. 2013). Since the extent of siRNA-mediated Lamin B1 depletion $(\sim 50-60 \%)$ was not as much as Lamin $\mathrm{A} / \mathrm{C}$ and $\mathrm{B} 2$ in our hands, we focused on examining the effects of Lamin $\mathrm{A} / \mathrm{C}$ and Lamin B2 knockdowns (Figs. $1 \mathrm{~b}$ and $\mathrm{S} 1 \mathrm{~b}$ ). Immunostaining showed $~ 85-90 \%$ depletion of Lamin A and B2 at the single-cell level (Fig. 1d, f). Counterstaining with Lamin B1 in Lamin A/C-depleted cells and 
a
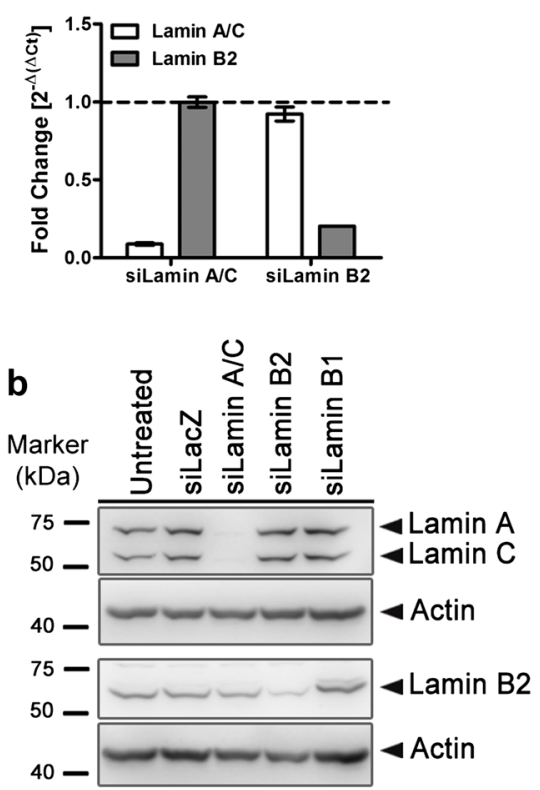

Fig. 1 Depletion of Lamin A/C and B2 in DLD1 cells. a qRT-PCR showing siRNA-mediated knockdown of Lamin A/C and Lamin B2 in DLD1 cells, normalized to expression levels of ACTIN and compared to nontargeting control (siLacZ). Data shown is a representative out of three independent biological replicates. Error bars represent standard error of mean (SEM). b Western blots showing knockdowns of Lamin A/C and Lamin B2 in DLD1 cells. Depletion of Lamin A/C does not impact the expression of Lamin B2 and vice versa. Loading control: actin. Data shown is a representative result from five independent experiments. c

Lamin A/C upon Lamin B2 Kd revealed distorted nuclear shapes, nuclear invaginations, blebbing, and nuclear furrows (Fig. S2) as found in Lamin A/C- and B1-depleted HeLa cells and in cells derived from laminopathies (Shimi et al. 2008; Taimen et al. 2009). Independently, Lamin A/C and B2 Kd were performed in DLD1 cells, followed by whole-genome expression profiling using gene expression arrays in three independent biological replicates. The data analyses revealed $\sim 300$ genes that were significantly deregulated (inclusive of upregulation and downregulation) in either Lamin A/C- or Lamin B2-depleted cells (absolute fold change $\geq 2.0$-fold) (Fig. 2a). Remarkably, in Lamin A/C Kd, 276 out of 298 genes were uniquely deregulated, while in Lamin B2 Kd, 279 out of 301 genes were uniquely deregulated (Fig. 2b). In both Lamin A/C- and B2-depleted cells, 22 genes $(\sim 7 \%)$ were commonly deregulated (Fig. 2b). Gene expression levels from expression arrays were independently validated using qRT-PCR for the top deregulated genes (absolute fold change $\sim 2-6$-fold). This showed a fold change in the same direction as that obtained from the microarray data set (Fig. S3a, b). We next performed a recovery assay where DLD1 cells were allowed to recover from the Lamin knockdowns by growing cells up to day 8 , well beyond the 48-h duration of the knockdown. At the end of day 8 , there was $\sim 60-70 \%$ recovery in the transcript and protein levels of Lamin A/C and B2, respectively (Fig. 2c-f). We detected a significant recovery in the transcript
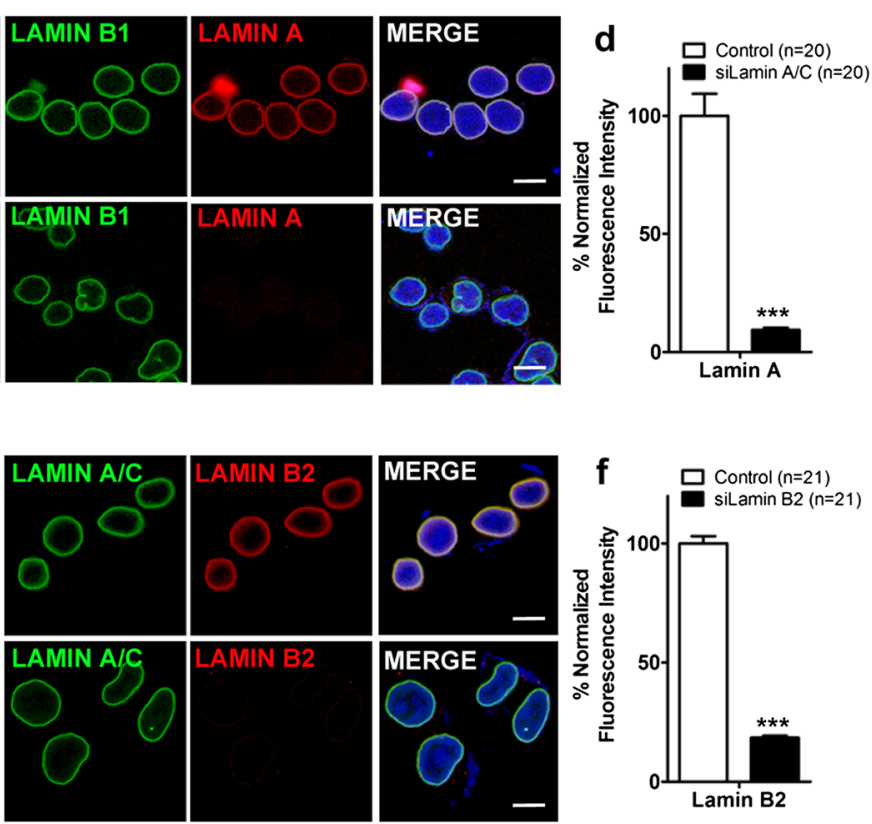

Immunostaining of Lamin B1 (green) and Lamin A (red) in control (untreated) and Lamin A/C Kd cells. Representative out of four independent experiments. Scale bar $\sim 10 \mu \mathrm{m}$. d Quantification of fluorescence intensity performed by line scans across nuclei from c. e Immunostaining of Lamin A/C (green) and Lamin B2 (red) in control (untreated) and Lamin B2 Kd cells. Scale bar $\sim 10 \mu \mathrm{m}$. f Quantification of fluorescence intensities performed by line scans across nuclei from e. Error bars in $\mathbf{d}, \mathbf{f}$ represent SEM, $n$ number of nuclei Knockdown of Lamin A and Lamin B2 was $\sim 85-90 \%$ at the single-cell level $(p<0.001$, unpaired Student's $t$ test)

levels of candidate genes $A B L I M 2$ and SMTNL2 upregulated in Lamin $\mathrm{A} / \mathrm{C} \mathrm{Kd}$ and TMEM14B and CDC42 downregulated in Lamin A/C Kd (Fig. 2g, h). Recovery was also detected for REG4 and PTGS2 upregulated in Lamin B2 Kd and MESDC2 and $\mathrm{KRCCl}$ downregulated upon Lamin B2 Kd (Fig. 2i, j).

Whole-genome expression profiling using microarrays from Lamin A mutant (E161K) human cardiomyocytes showed deregulated gene expression levels predominantly from gene-poor chromosome 13 (Mewborn et al. 2010). Likewise, mouse fibroblasts lacking functional Lamin B1 revealed transcriptionally deregulated clusters on the peripherally positioned mouse chromosome 18 (Malhas et al. 2007). These evidences prompted us to investigate if specific chromosomes were transcriptionally deregulated upon Lamin A/C or B2 depletion. From our expression array data sets independently derived from Lamin A/C and Lamin B2 knockdowns, we calculated the extent of transcriptional deregulation for each chromosome by expressing the total number of deregulated genes on a chromosome as a fraction of the total number of coding genes from that chromosome (Fig. 2k). Chromosomes showing $>0.7 \%$ deregulation in transcript levels were shortlisted. Chromosomes showing significant deregulation upon Lamin A/C Kd were Chr.16, 22, 20, 12, 1, 3, 17 , and 19 (descending order of expression deregulation). While upon Lamin B2 Kd, major deregulated chromosomes 


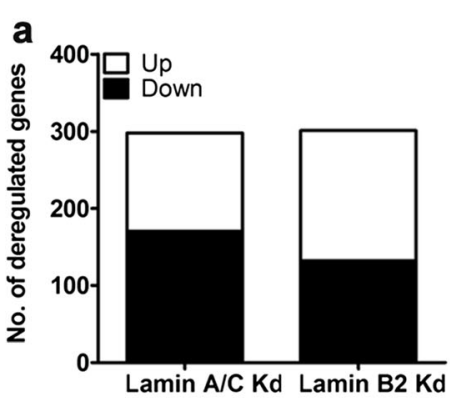

b Lamin A/C Lamin B2

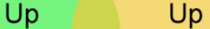

$110 \quad 14 \quad 154$

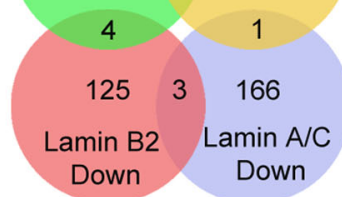

Lamin A/C Ko

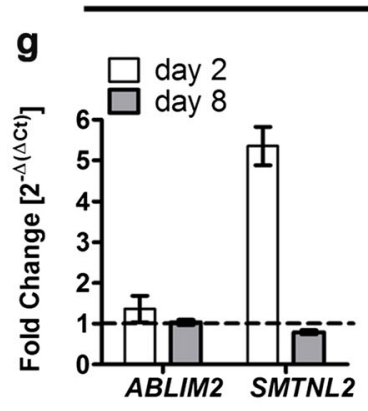

h

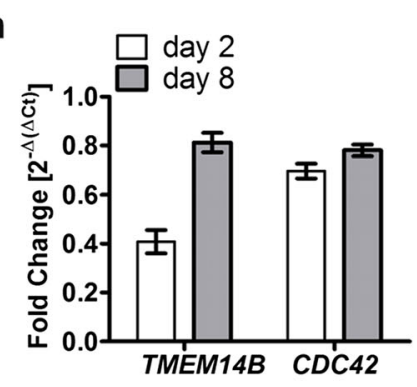

k

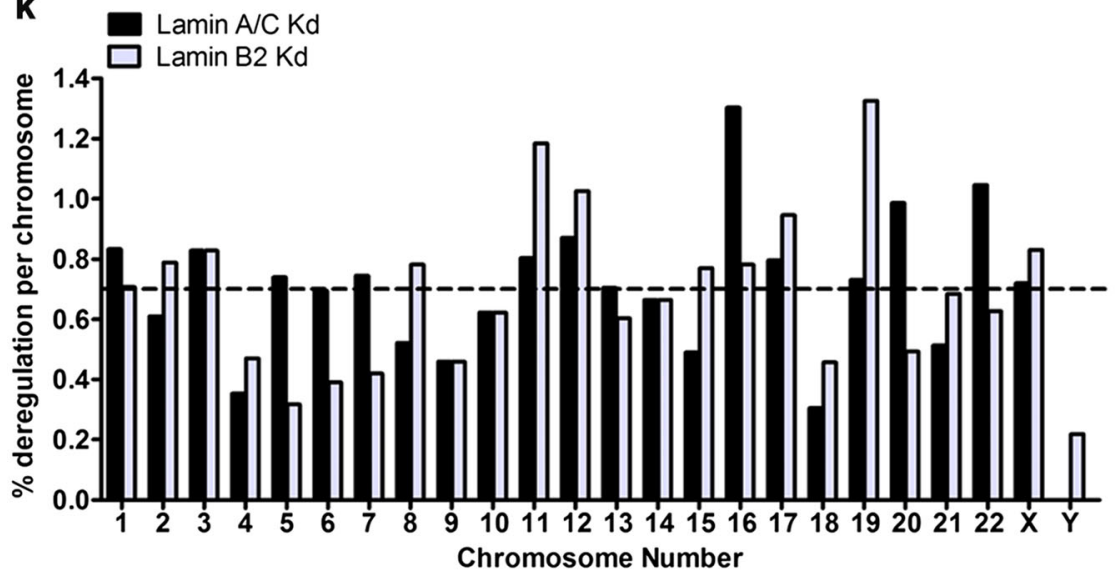

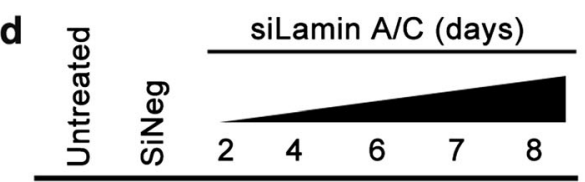

Lamin A $\triangle$ GAPDH
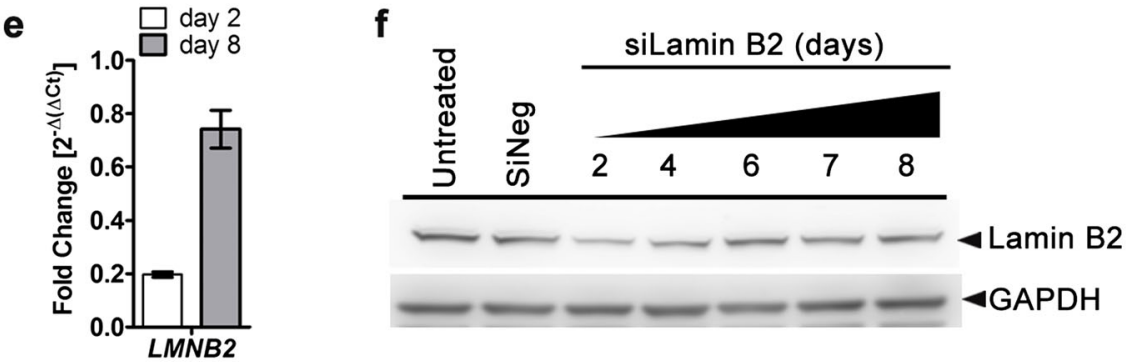
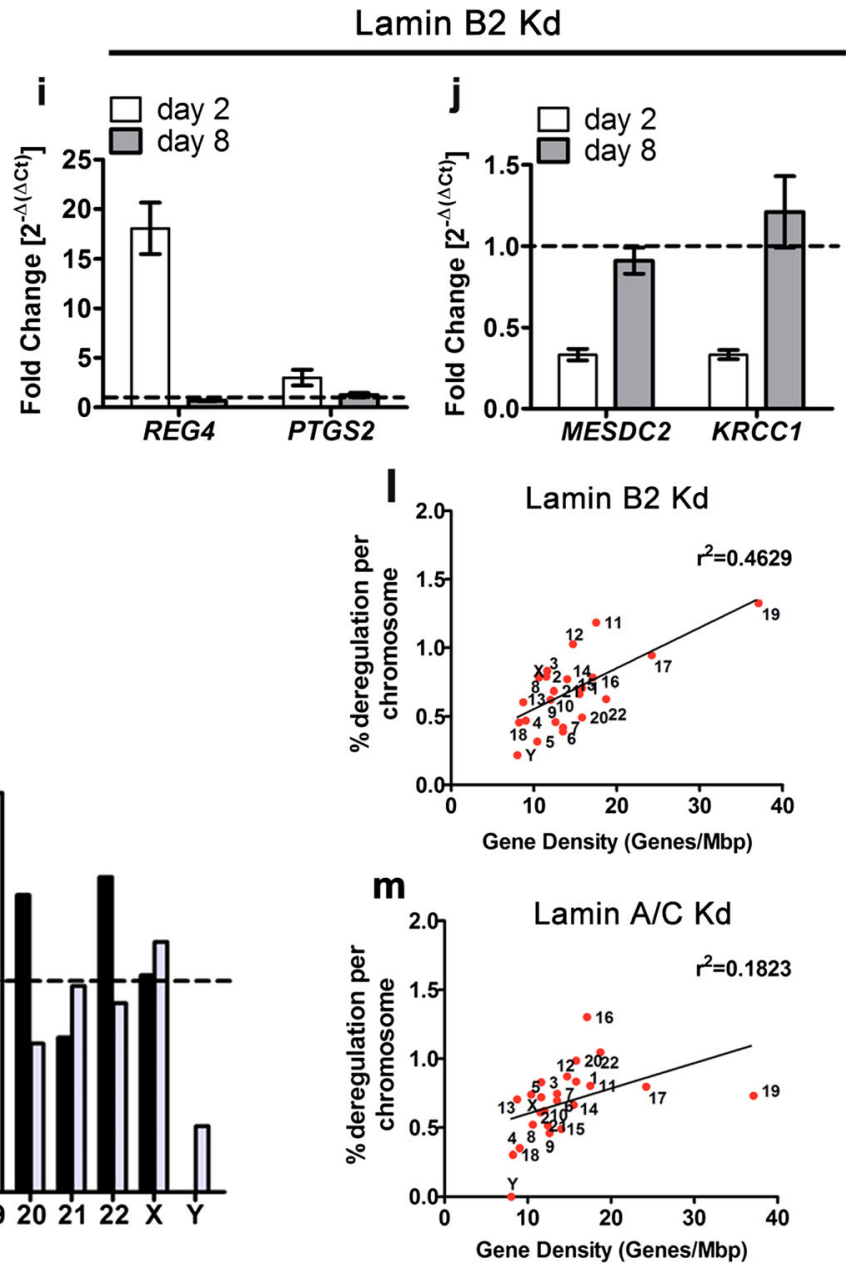

were Chr.19, 11, 12, 17, X, 8, 3, and 2 (descending order of expression deregulation) (Fig. 2k). Comparatively neither Lamin A/C Kd nor Lamin B2 Kd showed a significant transcriptional deregulation from gene-poor chromosomes 13, 18 or 7 (whole chromosomes), although these chromosomes are proximal to the nuclear lamina (Fig. 2k). A plot of 
4 Fig. 2 Impact of Lamin $\mathrm{A} / \mathrm{C}$ and $\mathrm{B} 2$ depletion on the transcriptome. a Number of deregulated genes (up and down) upon Lamin A/C and Lamin B2 depletion (cutoff $\geq 2$.0-fold on absolute scale) from genome-wide expression analyses. b Common and uniquely deregulated genes upon Lamin $\mathrm{A} / \mathrm{C}$ and Lamin $\mathrm{B} 2$ depletion. Lamin $\mathrm{A} / \mathrm{C}$ or $\mathrm{B} 2 \mathrm{Kd}$ reveals $\sim 300$ genes that were deregulated (Lamin A/C Kd-128 genes up, 170 genes down and Lamin B2 Kd-169 genes up, 132 genes down). c qRTPCR showing recovery of Lamin $\mathrm{A} / \mathrm{C}$ transcript at the end of 8 days, normalized to expression of ACTIN. d Western blot showing levels of Lamin A when DLD1 cells treated with siLamin A/C were grown for 2 8 days to assess recovery from siRNA-mediated knockdown. Loading control: GAPDH. e qRT-PCR showing recovery of Lamin B2 transcript at the end of 8 days, normalized to expression of ACTIN. f Western blot showing levels of Lamin B2 when DLD1 cells treated with siLamin B2 were grown for 2-8 days to assess recovery from siRNA-mediated knockdown. Loading control: GAPDH. Data shown in $\mathbf{d}, \mathbf{f}$ are representative results from two independent experiments. $g$ Expression levels of genes upregulated upon Lamin A/C Kd (ABLIM2, SMTNL2) at the end of day 2 were restored at day 8 . $\mathbf{h}$ Genes downregulated upon Lamin A/C $\mathrm{Kd}(T M E M 14 B, C D C 42)$ at the end of day 2 recovered at day 8 . i Expression levels of genes upregulated upon Lamin B2 Kd (REG4, $P T G S 2$ ) at the end of day 2 were restored at day 8. $\mathbf{j}$ Genes downregulated upon Lamin B2 $\mathrm{Kd}(M E S D C 2, K R C C 1)$ at the end of day 2 recovered at day 8. Data for all qRT-PCRs $(\mathbf{c}, \mathbf{e}, \mathbf{g}-\mathbf{j})$ is a compilation of two biological replicates and normalized to expression levels of ACTIN. Error bars represent SEM. k Genes deregulated per chromosome upon depletion of Lamin A/C and Lamin B2 in DLD1 cells. The \% deregulation= (no. of deregulated genes on a chromosome / total no. of coding genes on that chromosome) $\times 100$. Details of number of coding genes and size of chromosomes were obtained from MapViewer (NCBI). l, m Lamin B2 Kd $\left(r^{2}=0.4629\right)$ shows greater correlation between transcriptional deregulation and gene density as compared to Lamin $\mathrm{A} / \mathrm{C} \mathrm{Kd}\left(r^{2}=0.1823\right)$

$\%$ transcriptional deregulation against gene density showed a higher correlation with gene density upon Lamin B2 $\mathrm{Kd}$ $\left(r^{2}=0.4629\right)$ as compared to Lamin A/C Kd $\left(r^{2}=0.1823\right)$ (Fig. 21, m). This reveals an enhanced transcriptional deregulation from gene-rich chromosomes upon Lamin B2 Kd. Taken together, gene expression profiling of Lamin $\mathrm{A} / \mathrm{C}$ - or B2-depleted cells uncovered specific chromosomes that were significantly deregulated in their gene expression levels (Fig. 2k).

\section{Lamin depletion induces chromosomal aneuploidies}

We next performed three-dimensional fluorescence in situ hybridization (3D-FISH) of specifically those chromosomes that showed greater transcriptional deregulation over others independently in Lamin A/C and B2 depletion. Chromosome territories (CTs) 1 and 16 were examined upon Lamin $\mathrm{A} / \mathrm{C} \mathrm{Kd}$, and CTs 11 and 17 were examined upon Lamin B2 Kd (Fig. 3a, b). In addition, the most gene-rich chromosome 19 that was deregulated independently in Lamin A/C and B2 Kd was also examined along with gene-poor chromosome 18 of comparable DNA content (Fig. 3c). Furthermore, chromosome 18 showed a relatively lower $(\sim 0.5-0.6 \%)$ transcriptional deregulation in either Lamin $\mathrm{A} / \mathrm{C}$ or B2 $\mathrm{Kd}$ (Fig. 2k).
Three-dimensional FISH showed that Chr.1, 11, 16, 17, 18 , and 19 were diploid in $\sim 95 \%$ control cells (untreated cells and cells treated with non-targeting siRNA (siLacZ)) (Figs. 3d, e and $\mathrm{S} 4 \mathrm{a}-\mathrm{i}$ ). However, while imaging Lamin Kd nuclei, we consistently detected sub-populations ( $20-30 \%$ of cells) with more than two CTs (Fig. 3d, e). Chromosomes 18 and 19 were gained (three to four copies) in Lamin A/C Kd cells ( 20-30\%) (Figs. 3d and S4c, d), while chromosomes 11, 17, 18, and 19 were gained (three to four copies) in Lamin B2 knockdown cells (Figs. 3e and S4e-h). We also tested for the extent of aneuploidies of chromosomes that were not transcriptionally deregulated and therefore not shortlisted in each of Lamin A/C (CT11, 17) or B2 Kd (CT1, 16) cells. Chromosome 17 was gained (three copies) in Lamin A/ $\mathrm{C} \mathrm{Kd}$ in $\sim 20 \%$ cells, while chromosomes 1 and 16 were not gained upon Lamin B2 Kd (Fig. S4i). Immuno-FISH assays showed a comparable depletion of $\sim 75-80 \%$ of Lamin levels in both the diploid and aneuploid subpopulations of cells (Fig. S5). A scrambled (nontargeting) siRNA oligonucleotide control of Lamin B2 did not show aneuploidy for CT18 and 19, underscoring the specificity of the siRNA against Lamin B2 in DLD1 cells (Fig. S4j, k). In addition, an shRNA sequence against Lamin B2 showed a comparable extent of aneuploidy $(>30 \%)$ (data not shown), suggesting that Lamin-depletion-induced aneuploidies were simply not a manifestation of transient siRNA-mediated knockdowns.

We performed fluorescence-activated cell sorting (FACS) profiling to ascertain the ploidy of Lamin-depleted DLD1 cells (Fig. S6a-d). This profile showed a comparable proportion of cycling cells in the control and Lamin $\mathrm{Kd}$ populations. In addition, we did not detect polyploid sub-populations in control or Lamin Kd cells (Fig. S6a-d). Further, the levels of the major cell cycle checkpoint proteins Chk1 and Chk2 remained unaffected (Fig. S6e, f). To assess the effect of Lamin depletion on chromosomal instability (CIN), we examined metaphase spreads derived from Lamin knockdown cells (Fig. S7a-c). Control DLD1 cells showed a predominantly pseudo-diploid population ( $~ 80 \%$ ) (45-46 chromosomes) and another sub-population of $\sim 20 \%$ cells showing chromosomal losses and gains (Fig. S7d). An increase in the number of cells showing chromosomal losses (43-45 chromosomes) ( $p=0.0345)$ and gains (47-48 chromosomes) was detected upon Lamin A/C Kd (Fig. S7a, b, d). Remarkably, Lamin B2 Kd showed a significant increase in chromosomal gains (47-49 chromosomes) ( $p=0.018$ ) (Fig. S7a, c, d). This is consistent with chromosomal aneuploidies induced in DLD1 cells upon Lamin B2 depletion (Kuga et al. 2014). Karyotype analyses from metaphase spreads derived from control, Lamin $\mathrm{A} / \mathrm{C}$, or B2 Kd cells did not reveal any consistent chromosomal translocations (Fig. S7a-c). Notably, a comparable level of 

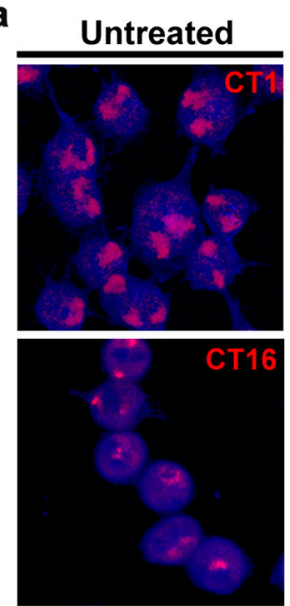

siLacZ
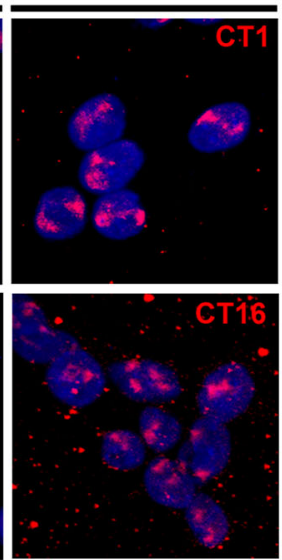

siLamin A/C
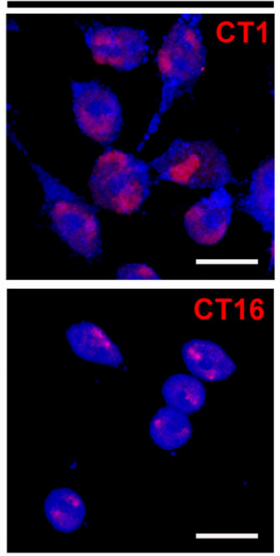

b
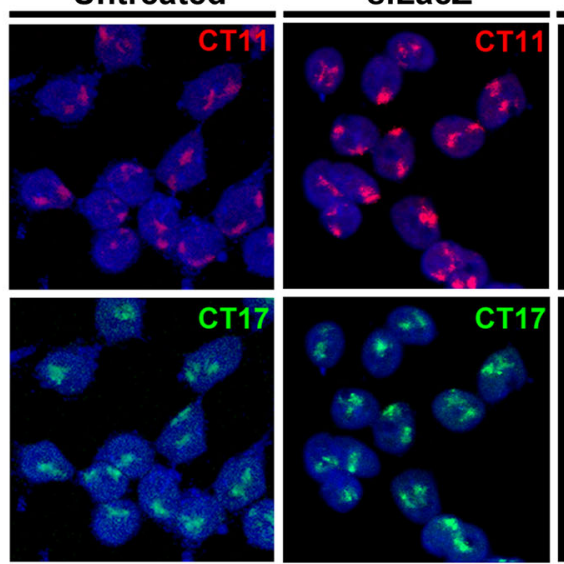

siLamin B2

C
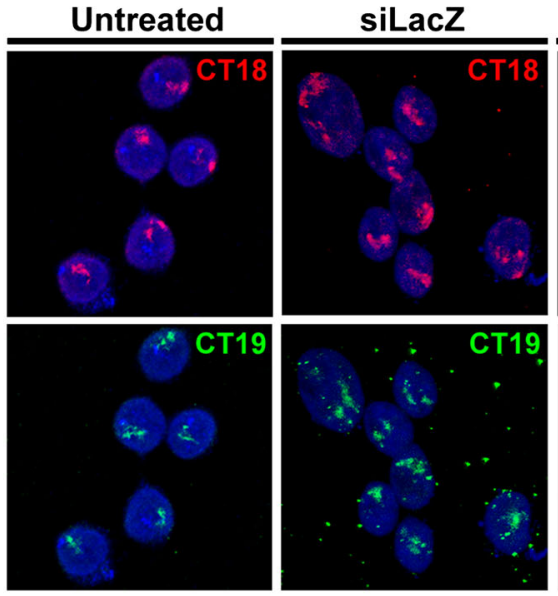

d

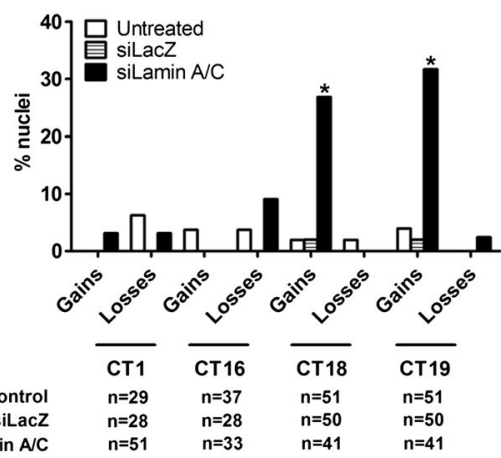

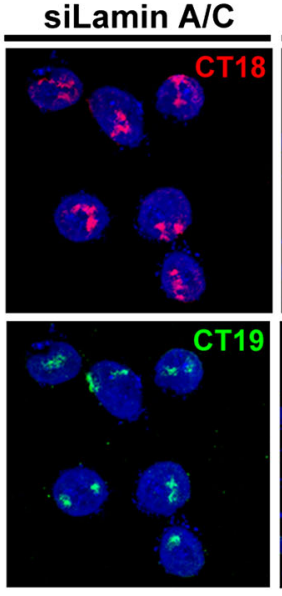

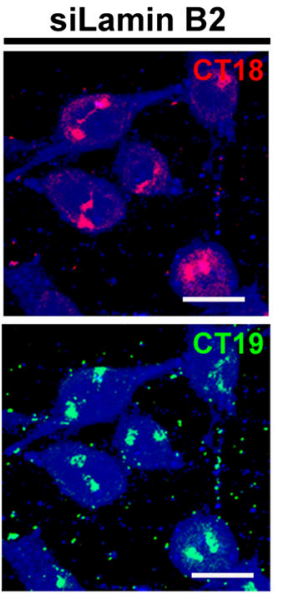

e

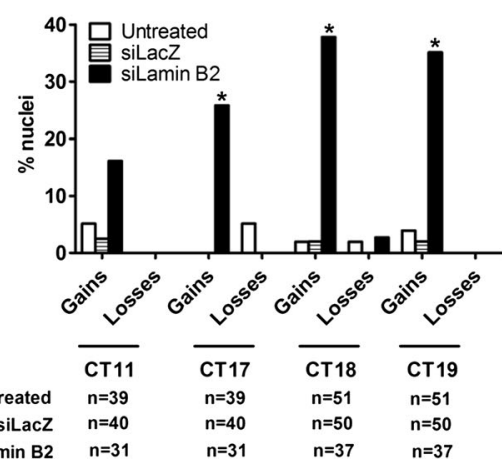

Fig. 3 Lamin depletion induces chromosomal aneuploidies. a Merged maximum intensity projection of confocal image stacks of chromosome territories (CT) - CT1 and CT16 for control (untreated and siLacZ treated) and siLamin A/C-treated DLD1 cells. b Merged maximum intensity projection of confocal image stacks of chromosome territories (CT) CT11 and CT17 for control (untreated and siLacZ treated) and siLamin B2treated DLD1 cells. c Merged maximum intensity projection of confocal image stacks of chromosome territories (CT) - CT18 and CT19 for control (untreated and siLacZ treated), siLamin A/C-, and siLamin B2-treated DLD1 cells. Nuclei are stained with DAPI, hybridizations for CT1 and CT16 were single-color hybridizations, while CT11-CT17 and CT18-

CIN ( $\sim 30 \%)$ is associated with colorectal cancer initiation and progression (Bardi et al. 1995; Ried et al. 1996).

Taken together, these studies show a specific induction of chromosomal aneuploidies upon Lamin $\mathrm{A} / \mathrm{C}$ and Lamin 
B2 depletion in DLD1 cells. We next examined the spatial organization of both the diploid and the aneuploid CTs in Lamin-depleted cells.

\section{Lamin $\mathrm{A} / \mathrm{C}$ depletion does not perturb conserved positions of diploid and aneuploid chromosome territories}

The spatial organization of chromosomes in the interphase nucleus largely correlates with gene density and, therefore, its expression levels, with gene-rich chromosomes in the nuclear interior being more transcriptionally active as against gene-poor chromosomes toward the nuclear periphery (Goetze et al. 2007). We performed 3D-FISH followed by confocal imaging and 3D radial distance measurements of diploid and aneuploid CTs in Lamin-depleted cells. The radial distance measurements have been represented both as a (i) dot scatter plots of raw data (Fig. S8) and (ii) binned into five shells of $\sim 20 \%$ of the nuclear sub-volume $(0 \%$-nuclear center, $100 \%$-nuclear periphery) (Figs. 4 and 5). It is pertinent to reiterate that the innermost nuclear shell enriched in euchromatin is relatively more transcriptionally active as compared to the peripheral shell $(\sim 80-100 \%)$ enriched in heterochromatin (Goetze et al. 2007). Three-dimensional FISH analyses of CT1 (gene density 15.83 genes/Mbp) and 16 (gene density 17.05 genes/Mbp) in Lamin A/C-depleted cells did not show a change in its radial distance distribution
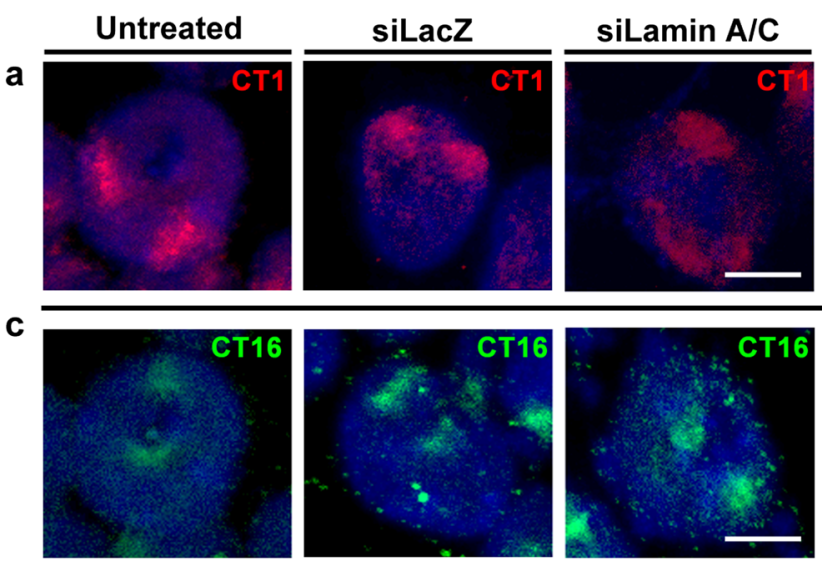
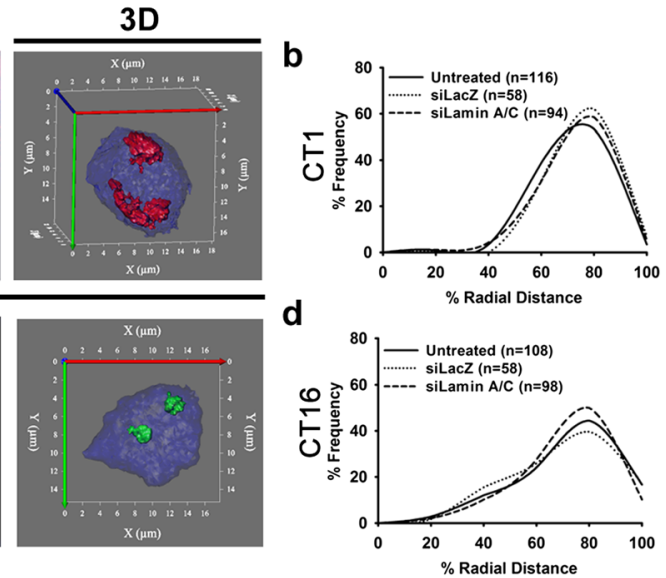

siLamin A/C
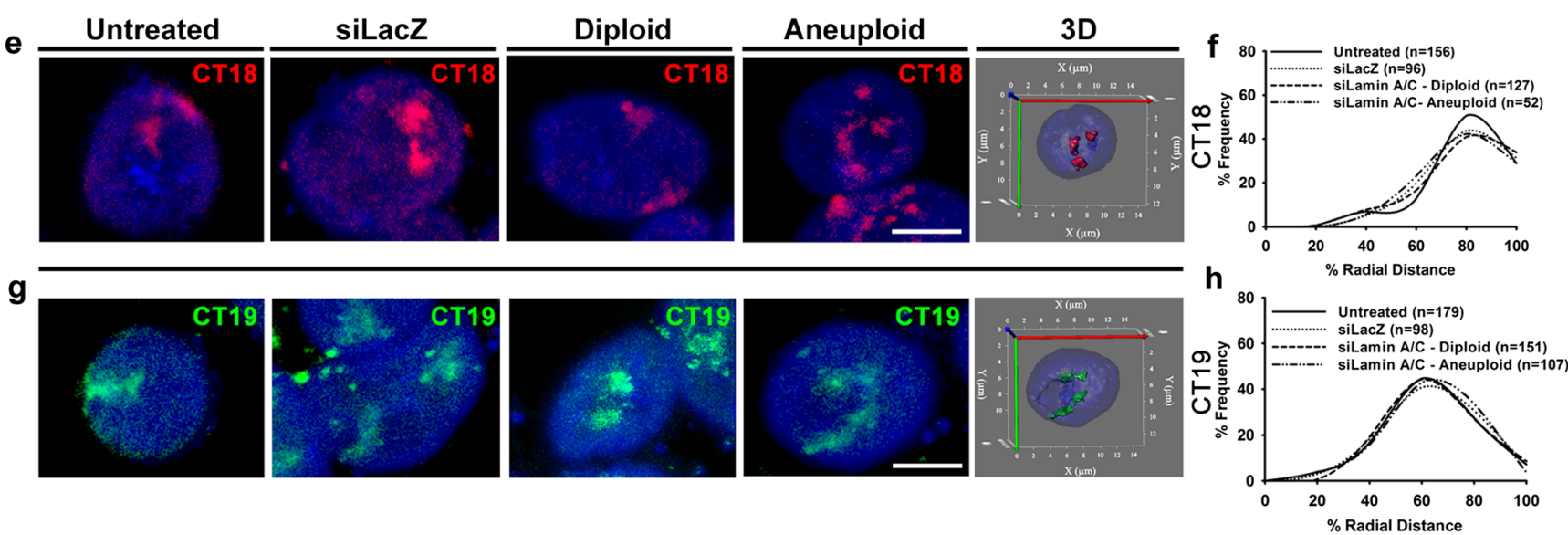

Fig. 4 Diploid and aneuploid chromosome territories assume conserved positions upon Lamin $\mathrm{A} / \mathrm{C}$ depletion. a-d Representative images and radial distance distribution profiles for 3D-FISH performed on untreated, siLacZtreated, and siLamin A/C-treated DLD1 cells for CT1 (a, b) and CT16 (c, d). Each panel shows merged maximum intensity projections of a representative nucleus from control (untreated, siLacZ) and siLamin A/C cells with its corresponding 3D reconstruction (3D). b, d Representative radial distance distribution profiles (pooled across experiments) of CT1 $(N=1)$ and CT16 $(N=2)$ binned into five sub-shells of $\sim 20 \%$ radial distance each. In each replicate, radial distances were measured for $\sim 50-100$ CTs. No significant change was detected in the radial distance distribution profiles of either diploid CT1 or CT16 upon Lamin A/C Kd $(p>0.05)$. e-h Merged maximum intensity projections for 3D-FISH images and radial distance distribution profiles for $\mathrm{CT} 18(\mathbf{e}, \mathbf{f})$ and $\mathrm{CT} 19(\mathbf{g}, \mathbf{h})$ which show aneuploidy upon Lamin A/C Kd. Representative 3D reconstructions show aneuploidy upon Lamin A/C Kd. f, h Radial distance distribution profiles (pooled across experiments) of CT18 $(N=2)$ and CT19 $(N=3)$ binned into five sub-shells of $\sim 20 \%$ radial distance each. In each replicate, radial distances were measured for $\sim 50-100$ CTs. No significant changes were detected in the distribution profiles for CT18 and CT19 in Lamin A/C Kd diploid or aneuploid cells $(p>0.05)$. Scale bar $\sim 5 \mu \mathrm{m}$. Fisher's exact test and $\chi^{2}$ test was used to test statistical significance of distribution in different sub-shells of the nucleus. $N$ number of independent experiments (biological replicates) contributing to the data, $n$ number of chromosome territories 


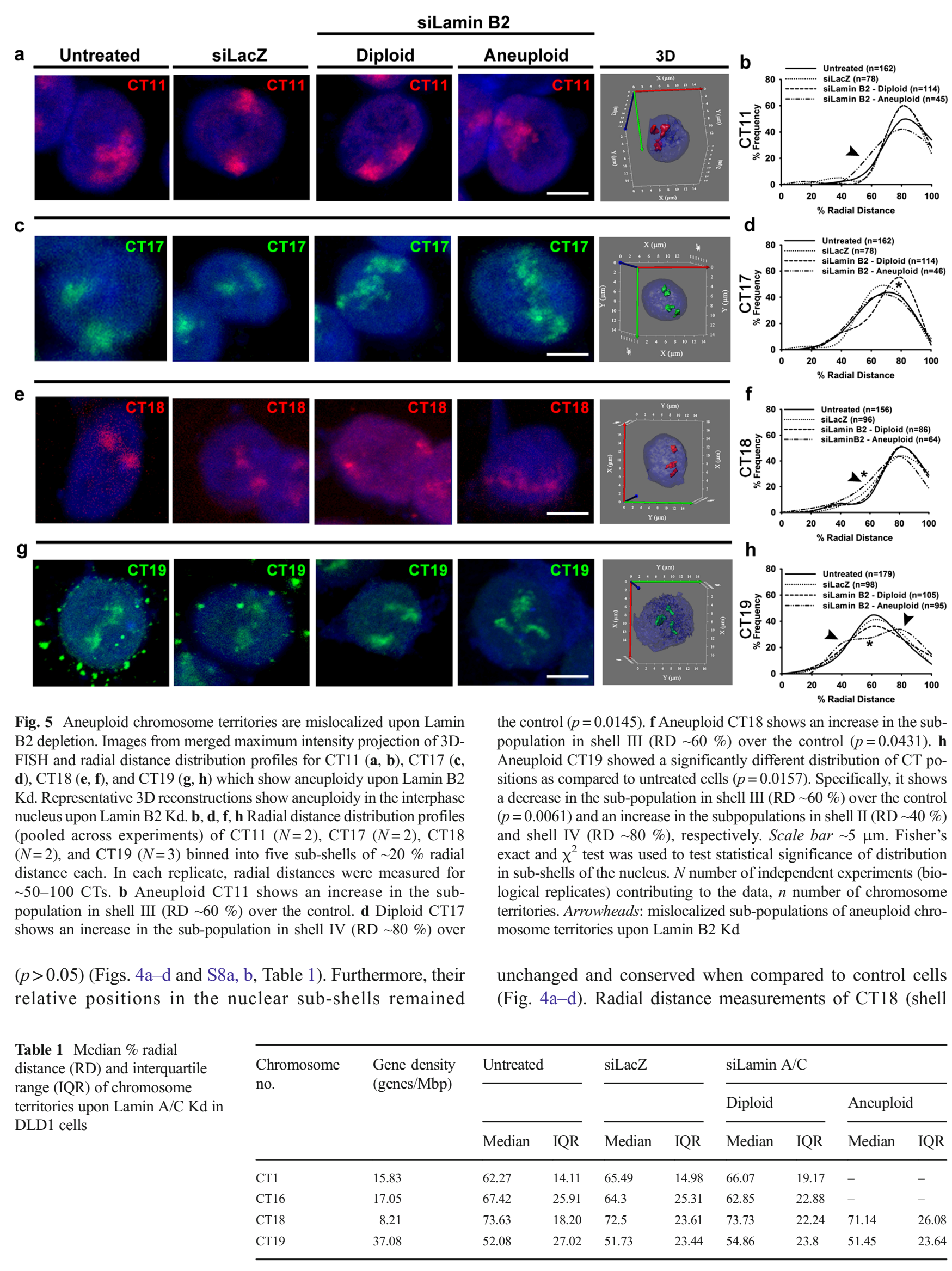


IV, RD $\sim 80 \%$ ) and CT19 (shell III, RD $\sim 60 \%$ ) in diploid or aneuploid cells remained conserved toward the nuclear periphery and nuclear interior, respectively (Figs. $4 \mathrm{e}-\mathrm{h}$ and S8c, d). Radial distance measurements of CT1, 16, 18, and 19 in the siLacZ-treated DLD1 cells remained conserved as compared to untreated control cells (Fig. 4b, d, f, h). In summary, despite the specific depletion of Lamin A/C, the diploid or aneuploid gene-rich or gene-poor CTs showed a remarkable conservation in their spatial localization consistent with their gene densities.

\section{Lamin B2 depletion mislocalizes aneuploid chromosome territories}

The increase in aneuploid cells upon Lamin B2 Kd is consistent with the known impact of Lamin B2 depletion in colorectal cancer cells (Kuga et al. 2014). We next analyzed the radial distance distributions of chromosomes that were transcriptionally deregulated upon Lamin B2 Kd in DLD1 cells. Although CT11 is gene rich ( $\sim 17.5$ genes/Mbp), it shows a peripheral (shell IV: RD $\sim 80 \%$ ) nuclear localization in control cells (Fig. 5a, b). Analyses of radial distance distribution of CT11 in Lamin B2 Kd cells showed a conserved radial distance distribution in diploid cells (shell IV: RD $\sim 80 \%$ ) (Fig. 5b). Aneuploid CT11 shows a mislocalized sub-population toward the nuclear interior to shell III: RD $\sim 60 \%$ (Fig. 5b). Interestingly, diploid CT17 (gene rich) showed a significant shift toward the nuclear periphery (shell IV: RD $\sim 80 \%$ ) as compared to control cells (shell III: RD $\sim 60 \%$ ) (Fig. 5 c, d). However, the aneuploid CT17 does not show a mislocalization as compared to control cells and remains clustered in shell III (RD 60\%) (Fig. 5d). It is plausible that the extra copy of CT17 may cluster into a single shell in Lamin B2 $\mathrm{Kd}$ cells, while one of the homologs in the diploid CT17 is mislocalized, suggesting differential effects that are exerted on CT17 in diploid and aneuploid Lamin B2 Kd cells - the functional relevance of which remains to be tested. The gene-poor and diploid CT18 in Lamin B2 Kd cells showed a predominantly peripheral nuclear localization (shell IV, RD $\sim 80 \%$ ). In addition, aneuploid CT18 shows an increase in a subpopulation of cells toward the nuclear interior (shell III, RD $\sim 60 \%$ ) (Fig. 5e, f). In contrast, the gene-rich CT19 predominantly localized toward the nuclear interior (shell III, RD $\sim 60 \%$ ) in control and diploid cells depleted in Lamin B2. However, aneuploid CT19 showed a striking mislocalization in its distribution into nuclear sub-shells, repositioning further toward the nuclear interior (shell II: RD $\sim 40 \%$ ) and nuclear periphery (shell IV: RD $\sim 80 \%$ ) (Fig. 5g, h). Radial distance measurements of CT11, 17, 18, and 19 in the siLacZ-treated DLD1 cells remained conserved as compared to untreated control cells (Fig. 4b, d, f, h). Taken together, aneuploid CTs show mislocalized sub-populations upon Lamin B2 depletion. We examined the interquartile range (IQR) of the radial distance distributions of the CTs, which consistently showed a greater IQR (22.73-31.19) for the aneuploid cells than for the diploid cells (11.21-29.97) (Table 2).

\section{Spatial organization of gene loci is altered upon Lamin B2 depletion}

We show that Lamin B2 depletion mislocalizes aneuploid CTs. We therefore examined if the mislocalization of aneuploid CTs also impinges on the spatial organization and function of a target gene (Fig. 6). We examined the role of Lamin B2 in regulating gene expression of a candidate gene on chromosome 19. The spatial organization of gene loci in the interphase nucleus is non-random and correlates with its expression levels. Gene loci that are overexpressed typically loop-out of their respective CTs (Chambeyron and Bickmore 2004; Volpi et al. 2000). Furthermore, gene loci associate with nuclear landmarks such as the nuclear lamina, Polycomb bodies, which further impact its transcription status (Bracken et al. 2006; Guelen et al. 2008). The nuclear lamina is predominantly a transcriptionally repressive zone, and inactive genes typically associate with nuclear Lamins at the nuclear periphery in contrast to active genes (Guelen et al. 2008; Harr et al. 2015; Peric-Hupkes et al. 2010; Reddy et al. 2008).

We adopted the nuclear lamina as a reference for examining the spatial organization of the gene locus in 3D (Reddy et al. 2008; Zullo et al. 2012) (Fig. 6a). Since we
Table 2 Median \% radial distance $(\mathrm{RD})$ and interquartile range (IQR) of chromosome territories upon Lamin B2 $\mathrm{Kd}$ in DLD1 cells

\begin{tabular}{|c|c|c|c|c|c|c|c|c|c|}
\hline \multirow{3}{*}{$\begin{array}{l}\text { Chromosome } \\
\text { no. }\end{array}$} & \multirow{3}{*}{$\begin{array}{l}\text { Gene density } \\
\text { (genes/Mbp) }\end{array}$} & \multicolumn{2}{|c|}{ Untreated } & \multicolumn{2}{|l|}{ siLacZ } & \multicolumn{4}{|c|}{ siLamin B2 } \\
\hline & & \multirow[b]{2}{*}{ Median } & \multirow[b]{2}{*}{ IQR } & \multirow[b]{2}{*}{ Median } & \multirow[b]{2}{*}{ IQR } & \multicolumn{2}{|l|}{ Diploid } & \multicolumn{2}{|c|}{ Aneuploid } \\
\hline & & & & & & Median & IQR & Median & IQR \\
\hline CT11 & 17.5 & 76.19 & 17.25 & 73.55 & 16.81 & 75.06 & 11.21 & 73.11 & 22.73 \\
\hline CT17 & 24.21 & 57.94 & 24.53 & 57.79 & 18.84 & 62.22 & 14.69 & 57.6 & 27.87 \\
\hline CT18 & 8.21 & 73.63 & 18.20 & 72.5 & 23.61 & 70.45 & 19 & 69.25 & 23.3 \\
\hline CT19 & 37.08 & 52.08 & 27.02 & 51.73 & 23.44 & 53.88 & 29.97 & 57.23 & 31.19 \\
\hline
\end{tabular}


Fig. 6 Enhanced expression level and altered spatial organization of gene loci upon Lamin B2 depletion. a Representative images (from a single optical section of a confocal image stack) of 3D Immuno-FISH for Lamin A (green), Lamin B2 (cyan), ZNF570 (red), and DAPI (blue) performed on siLacZ- and siLamin B2-treated DLD1 cells. Scale bar $\sim 5 \mu \mathrm{m}$. b qRT-PCR showing upregulation of ZNF570 upon Lamin B2 Kd in DLD1

cells. Data shown is a representation out of two independent experiments Error bars represent SEM. c Dot scatter plot showing least distance between ZNF570 locus and Lamin A signal in siLacZ- and siLamin B2-treated DLD1 cells, horizontal bar represents median. In each of siLacZ and siLamin B2 cells, $\sim 60-70$ gene loci signals were quantified. The distance of ZNF570 locus with respect to the lamina was significantly greater in the siLamin B2 cells $(p=0.004, \mathrm{~K}-\mathrm{S}$ test $)$.

Arrowheads: ZNF570 gene locus a

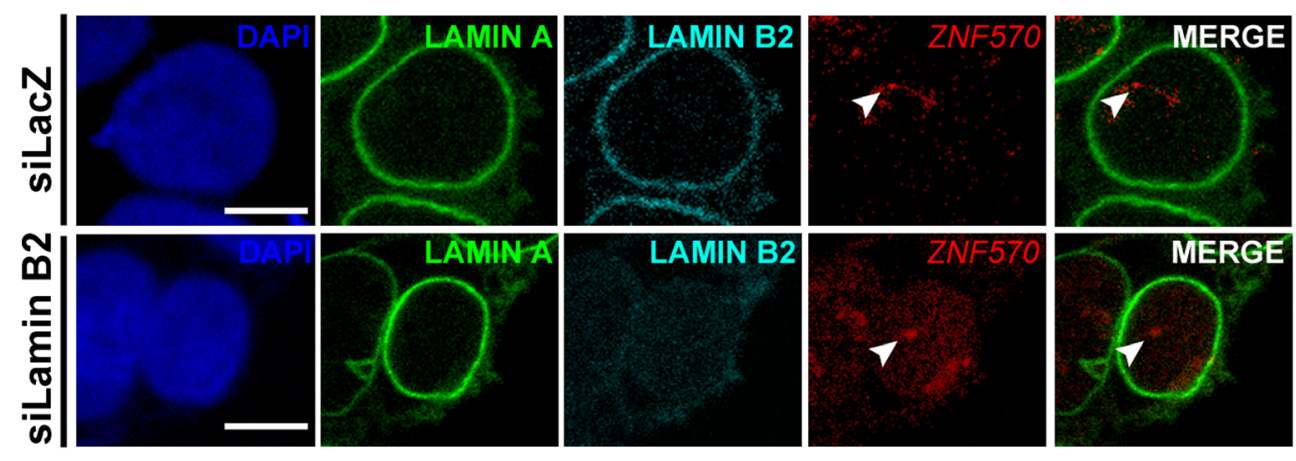

b

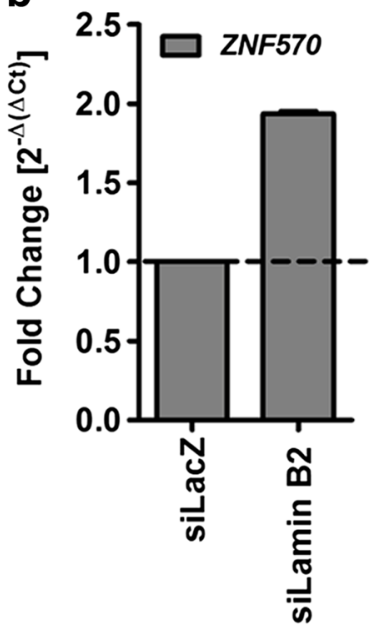

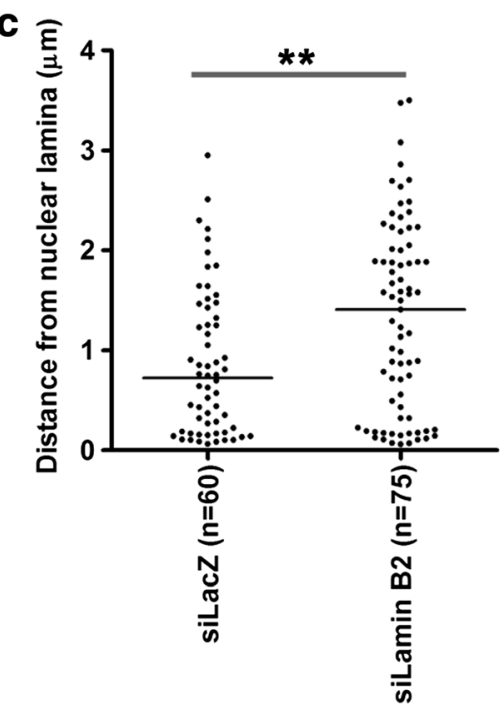

consistently detected chromosome 19 aneuploidies upon Lamin B2 knockdown, we examined the spatial organization of a candidate gene locus ZNF570 (Chr.19q13.12) (Fig. 6a). The ZNF570 gene locus was also found to be aneuploid in $\sim 25 \%$ Lamin B2-depleted cells. Gene expression profiling using microarrays showed a $\sim 2.96$-fold upregulation of ZNF570, which was corroborated by qRTPCR ( 2-fold) (Fig. 6b). We measured the distance of the fluorescently labeled ZNF570 gene locus with respect to the nuclear lamina (Lamin A) that we considered as the origin, i.e., $0 \mu \mathrm{m}$ (Fig. 6c). The ZNF570 gene locus showed a predominant position proximal to the nuclear lamina (median $=0.72 \mu \mathrm{m}$ ) in control cells (siLacZ) and showed a significant shift away from the nuclear lamina upon Lamin B2 depletion (median $=1.40 \mu \mathrm{m}$ ) (Fig. 6c). The spatial organization of ZNF570 showed a similar repositioning away from the nuclear lamina in diploid and aneuploid Lamin B2 Kd cells (Fig. S9a, b). Furthermore, we detected a significant increase in the number of RNA transcript signals for ZNF570 in Lamin B2-depleted cells (Fig. S9c, d). Thus, a loss of Lamin B2 repositions ZNF570 gene locus (diploid and aneuploid) away from the nuclear lamina, which further manifests as an increase in transcript levels detected in single cells (RNA-FISH) as well as at the population level (qRT-PCR).

\section{Chromosome territory volumes are altered in Lamin-depleted cells}

Lamin A mutation $(\mathrm{E} 161 \mathrm{~K})$ showed a decrease in the volume of chromosome 13 territory in human fibroblasts (Mewborn et al. 2010). Volumes of chromosome 18 and 19 territories showed an increase in Lamin B1-depleted DLD1 cells (Camps et al. 2014). We next examined if Lamin A/C or B2 depletion affects the volumes of CTs in either diploid or aneuploid cells (Fig. 7, Tables 3 and 4). The nuclear volume showed a significant increase ( 1.1-fold) upon Lamin A/C Kd (Fig. 7a). Diploid chromosome 1, 18, and 19 territories showed a significant increase in their volumes $(\sim 1.2$-fold) upon Lamin $\mathrm{A} / \mathrm{C}$ depletion (Fig. 7b, d, e). Chromosome 16 did not show a change in its volume (Fig. 7c). Aneuploid CT18 and CT19 did not show a significant change in volume upon Lamin A/C Kd (Fig. 7d, e). This suggests a role for Lamin $\mathrm{A} / \mathrm{C}$ in either directly or indirectly regulating $\mathrm{CT}$ volumes, primarily of diploid CTs. 
Lamin B2 depletion shows an 1 .2-fold increase in nuclear volume (Fig. 7f). CT11 and CT17 did not show a change in volume in either diploid or aneuploid state (Fig. $7 g, h)$. CT18 showed an increase in volumes ( 1.7-fold) in the aneuploid state, and CT19 showed an increase in volume in the diploid ( 1.4fold) as well as aneuploid state ( 1.5-fold) (Fig. 7i, j). In summary, Lamin A/C and B2 depletion impacts nuclear volumes. While Lamin A/C Kd primarily affects volumes of diploid CTs, Lamin B2 Kd impacts volumes of mainly aneuploid CTs.

To further probe if mislocalized aneuploid CTs show an altered volume upon Lamin B2 $\mathrm{Kd}$, we examined the volumes of aneuploid CTs across concentric nuclear subshells (Fig. 7g'-j'). We did not detect a significant difference in the volumes of aneuploid CTs whether they were localized toward the nuclear interior or toward the nuclear periphery, further suggesting that the altered volume of aneuploid CTs tested here (CT18, CT19) may not correlate with its mislocalization into a different nuclear subshell.

\section{Lamin depletion destabilizes spatial constraints that confine chromosome territories into distinct nuclear sub-volumes}

We examined the spatial organization of both diploid and aneuploid CTs in Lamin-depleted cells. Lamin depletion showed an increase in nuclear volume of $\sim 1.1$ fold in Lamin A/C Kd cells and 1 .2-fold in Lamin B2 $\mathrm{Kd}$ cells. A spherical nucleus can be grossly subdivided into five concentric sub-shells, with the generich CTs predominantly toward the nuclear interior (shell II-III), while gene-poor CTs are toward the nuclear periphery (shell IV-V) (Fig. 8a). Chromosomal aneuploidies generated upon Lamin A/C and B2 depletion generate diploid and aneuploid chromosomal subpopulations. Diploid CTs in either Lamin A/C- or B2depleted cells show conserved positions in a genedensity-dependent manner. However, Lamin A/C knockdown cells showed an increase in CT volume in the diploid sub-population of cells (Fig. 8b). In contrast, aneuploid CTs in Lamin B2-depleted cells are strikingly mislocalized and show altered volumes in the interphase nucleus (Fig. 8c). Our results strongly implicate the presence of additional underlying Lamin B2-dependent mechanisms that facilitate gene-density-based chromosome positioning in the interphase nucleus. This potentially includes (i) direct associations of Lamin B2 with chromatin and (ii) protein-protein interactomes of Lamin B2 or a combination of both these factors. Taken together, our studies highlight a unique role for Lamin B2 in regulating the spatial localization of aneuploid CTs in the interphase nucleus.

\section{Discussion}

Lamins are required for the maintenance of nuclear structure and function. Furthermore, Lamins are necessary for the appropriate localization of CTs in the interphase nucleus (Malhas et al. 2007; Mewborn et al. 2010; Shimi et al. 2008). Our results suggest a requirement for Lamin B2 since its absence results in mislocalization of aneuploid CTs. The specific role of Lamin B2 in regulating chromosomal ploidy and chromosome positioning is difficult to uncouple. While both A- and B-type Lamins are involved in regulating spindle assembly during mitosis (Goodman et al. 2010; Kuga et al. 2014; Qi et al. 2015; Tsai et al. 2006), Lamins are primarily structural proteins that maintain the spatial organization of the genome (Peric-Hupkes and van Steensel 2010). While both Lamin A/C and Lamin B2 depletion induced CIN, primarily Lamin B2 depletion resulted in a mislocalization of aneuploid CTs. There are conflicting reports on the expression levels of Lamin A/C in colorectal cancers (Belt et al. 2011; Foster et al. 2011; Moss et al. 1999; Willis et al. 2008; Wu et al. 2009). In contrast, Lamin B2 was shown to be a differentially expressed protein between colorectal cancers with and without CINwith increase in Lamin B2 expression conferring protection against CIN (Kuga et al. 2014). It is noteworthy from our results that Chr.17, 18, and 19 (from the specific subset of chromosomes that we examined) were consistently gained independently upon Lamin A/C or Lamin B2 depletion (Fig. S4i). This is suggestive of a pattern of chromosomal acquisitions potentially relevant for colorectal cancer progression (Tagawa et al. 1996). It remains to be examined if the extent of Lamin stoichiometry correlates with the extent of these chromosomal gains during colorectal cancer progression (Belt et al. 2011; Roth et al. 2010).

Our study highlights an interesting divergence of roles for Lamin A/C and Lamin B2. The role of Lamin B2 in maintenance of genome organization is rather underappreciated. Diploid DLD1 cells robustly express Lamins A/C, B1, and B2 that potentially compensate for one another in single Lamin knockdowns in order to maintain conserved chromosome positions. This is further reiterated in Lamin B1depleted DLD1 cells that show a conserved localization of CT18 at the nuclear periphery (Camps et al. 2014). However, in aneuploid cells, an optimum level of Lamin B2 is a likely prerequisite for the correct spatial positioning of CTs. It is plausible that regulation of ploidy and positioning of aneuploid chromosomes are co-regulated by Lamin B2 through its commonly regulated interactors that function during both mitosis and interphase. One such candidate is the inner nuclear membrane protein SUN1 identified in a screen by Kuga et al. (2014) which associates with Lamin B2 during mitosis. However, the precise roles of Lamin B2-associated interactors in the context of maintaining the spatial organization of the genome is unclear. 

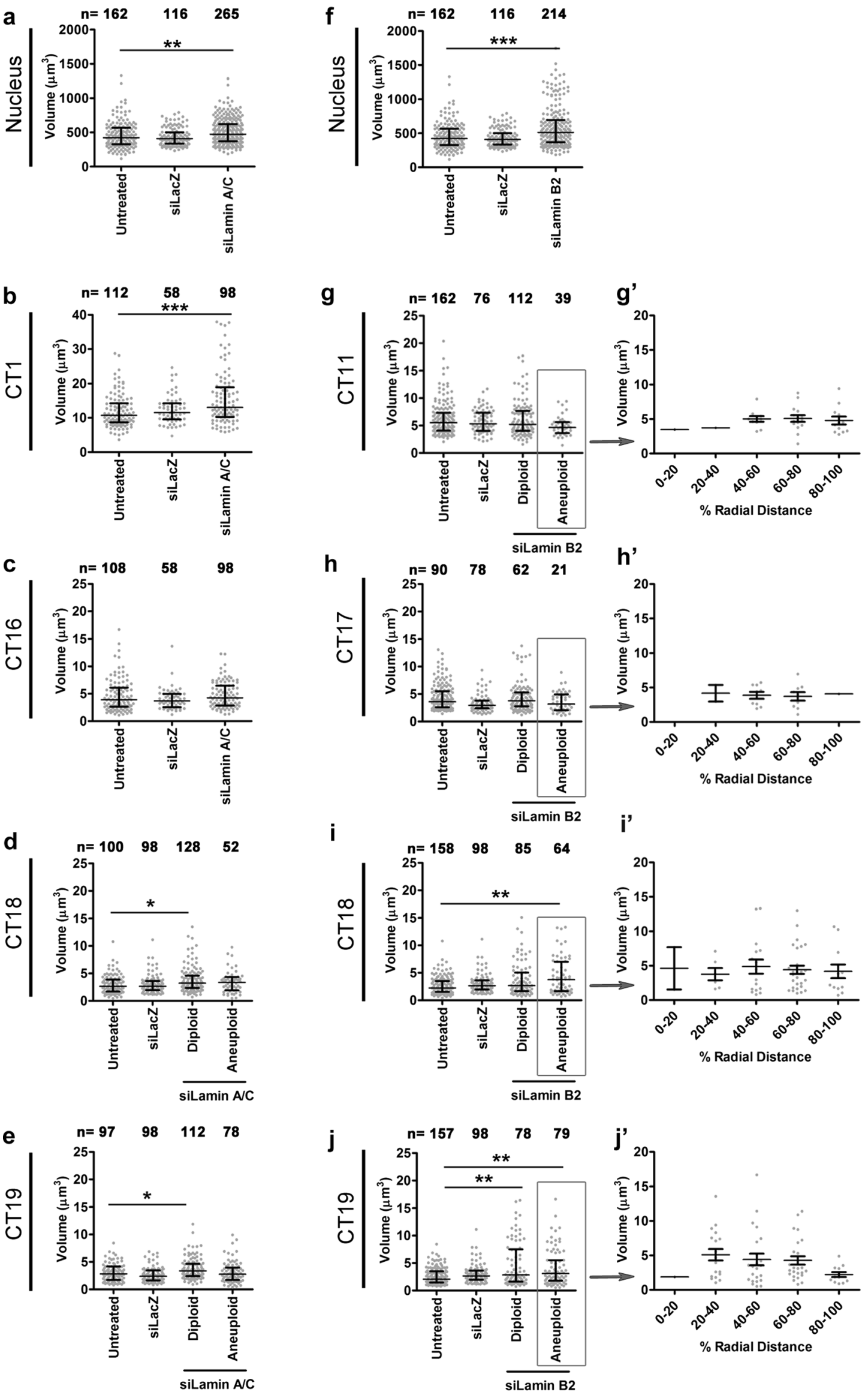
4 Fig. 7 Chromosome territories show volume changes upon Lamin depletion. $\mathbf{a}-\mathbf{j}$ Dot scatter plots for volumes of nuclei and chromosome territories upon Lamin A/C and Lamin B2 depletion in DLD1 cells. Horizontal bars indicate median with the interquartile range. a A significant increase was detected for the nuclear volume ( 1.1-fold) upon Lamin $\mathrm{A} / \mathrm{C} \mathrm{Kd}(p=0.0001), N=5$. b A significant increase was detected for volumes of CT1 $(p=0.0009), N=1$ upon Lamin A/C Kd. c No significant difference in volume was detected for $\mathrm{CT} 16, N=2$. d, e A significant increase in the volume of diploid CT18 $(p=0.0149), N=2$, and CT19 $(p<0.0001), N=2$, while aneuploid CT18 and CT19 do not show a significant difference in volume upon Lamin A/C Kd. f Nuclear volume shows a significant increase upon Lamin B2 Kd ( 1.2-fold, $p<0.0001)$, $N=5 . \mathbf{g}, \mathbf{h}$ No significant difference in volume for either diploid or aneuploid CT11 $(N=2)$ or CT17 $(N=1)$ upon Lamin B2 Kd. i A significant increase in the volume of aneuploid CT18 $(p=0.0019), N=2$. j Significant increase in the volume of diploid and aneuploid CT19 upon Lamin B2 Kd $(p=0.0004), N=2$. $\mathbf{g}^{\prime}-\mathbf{j}$ ' Distribution of the volumes of aneuploid chromosome territories (CT11, CT17, CT18, and CT19) across nuclear sub-shells upon Lamin B2 depletion. No significant difference was detected in the volumes of aneuploid CT across nuclear sub-shells. Kruskal-Wallis test was used to test for statistical significance. $N$ number of independent experiments (biological replicates) contributing to the data, $n$ number of nuclei $(\mathbf{a}, \mathbf{f})$ or chromosome territories $(\mathbf{b}-\mathbf{e}, \mathbf{g}-\mathbf{j})$ scored

Previous studies suggest a remarkable conservation in gene-density-based chromosome positioning patterns of even aneuploid chromosomes in the interphase nucleus in cancer cells (Cremer et al. 2003). A recent study on primary amniocyte cells shows that naturally aneuploid human Chr.18 and 21 territories assume conserved nuclear locations (Hervé et al. 2016). Further, artificially introduced gene-poor human Chr.7 or 18 and gene-rich Chr.19 into otherwise diploid DLD1 cells showed a gene-density-based conservation in their positioning patterns toward the nuclear periphery and nuclear interior, respectively (Sengupta et al. 2007). It is therefore likely that Lamins play a crucial role in the conservation of spatial organization of aneuploid (trisomies) CTs. It is conceivable that Lamin depletion destabilizes different subsets of Lamin interaction networks at the (i) nuclear periphery (linker of nucleoskeleton and cytoskeleton (LINC) complex), (ii) heterochromatin, (iii) nuclear interior, and (iv) perinucleolar heterochromatin (Dechat et al. 2010; Gruenbaum and Medalia 2015). At the nuclear periphery, Lamins interact with LEM-

Table 3 Median volume of chromosome territories upon Lamin A/C $\mathrm{Kd}$ in DLD1 cells

\begin{tabular}{lcccl}
\hline $\begin{array}{l}\text { Chromosome } \\
\text { no. }\end{array}$ & Untreated $\left(\mu \mathrm{m}^{3}\right)$ & $\operatorname{siLacZ}\left(\mu \mathrm{m}^{3}\right)$ & \multicolumn{2}{c}{$\operatorname{siLamin} \mathrm{A} / \mathrm{C}\left(\mu \mathrm{m}^{3}\right)$} \\
\cline { 3 - 5 } & & & Diploid & Aneuploid \\
\hline CT1 & 10.73 & 11.51 & $13.05^{*}$ & - \\
CT16 & 3.89 & 3.69 & 4.26 & - \\
CT18 & 2.65 & 2.65 & $3.21^{*}$ & 3.36 \\
CT19 & 2.83 & 2.39 & $3.37^{*}$ & 2.75 \\
\hline
\end{tabular}

*Significant $(p<0.05)$
Table 4 Median volume of chromosome territories upon Lamin B2 Kd in DLD1 cells

\begin{tabular}{lllll}
\hline \multirow{2}{*}{$\begin{array}{l}\text { Chromosome } \\
\text { no. }\end{array}$} & Untreated $\left(\mu \mathrm{m}^{3}\right)$ & siLacZ $\left(\mu \mathrm{m}^{3}\right)$ & \multicolumn{2}{l}{ siLamin B2 $\left(\mu \mathrm{m}^{3}\right)$} \\
\cline { 4 - 5 } & & & Diploid & Aneuploid \\
\hline CT11 & 5.53 & 5.32 & 5.22 & 4.64 \\
CT17 & 2.99 & 2.94 & 3.32 & 4.07 \\
CT18 & 2.23 & 2.65 & 2.66 & $3.76^{*}$ \\
CT19 & 2.06 & 2.65 & $2.84^{*}$ & $3.08^{*}$ \\
\hline
\end{tabular}

*Significant $(p<0.05)$

D proteins, LBR, and HP1 involved in heterochromatin organization (Clements et al. 2000; Foisner and Gerace 1993; Smith and Blobel 1994; Solovei et al. 2013). We surmise that Lamin B2 depletion results in a general weakening of the nuclear periphery due to a loss of its association with Lamin $\mathrm{A} / \mathrm{C}$ and other factors such as LINC complex, LBR (Moir et al. 2000; Shimi et al. 2015; Ye et al. 1997). This not only maintains the integrity of the nuclear periphery but also tethers gene-poor CTs since aneuploid CT18 (in Lamin B2-depleted cells) disengages from the nuclear lamina and moves further into the nuclear interior. In the nucleoplasm, Lamins associate with chromatin organizers - CTCF, BANF1, and Laminaassociated polypeptide (LAP $2 \alpha / \beta)$ that assist chromatin organization (Dechat et al. 2000; Gesson et al. 2016; Yusufzai et al. 2004). This is likely to impinge on the spatial organization of gene-rich CTs such as CT19 toward the nuclear interior. The role of phosphorylated Lamin A (in the nuclear interior as demonstrated in HeLa cells) (Kochin et al. 2014) in genome organization is unclear. Lamin $\mathrm{A} / \mathrm{C}$ and $\mathrm{LBR}$ are required for tethering heterochromatin, while its absence results in a mislocalization of heterochromatin to the nuclear interior and, therefore, an inversion in nuclear architecture in murine rod cells (Solovei et al. 2009, 2013). Our studies suggest a similar alteration in genome organization as evidenced by the mislocalization of the gene-rich CT19 toward the nuclear periphery and gene-poor CT18 toward the nuclear interior specifically in Lamin B2-depleted aneuploid cells (Fig. 5f, h). This suggests a requirement specifically for Lamin B2 and its interactome in maintaining conserved CT positions. A careful biochemical analysis of these nuclear sub-fractions may provide further insights on these potential interactions with Lamin B2 required for the correct maintenance of CTs in a manner consistent with gene density. Alternatively, the decision to place chromosomes in unique nuclear locations is likely to be initiated during mitosis, where Lamins regulate chromosome segregation (Martin et al. 2010).

It is pertinent to note that Lamin-depletion-induced volume changes of CTs may further impinge on the spatial constraints that position CTs and their transcription potential (Fig. 7). 


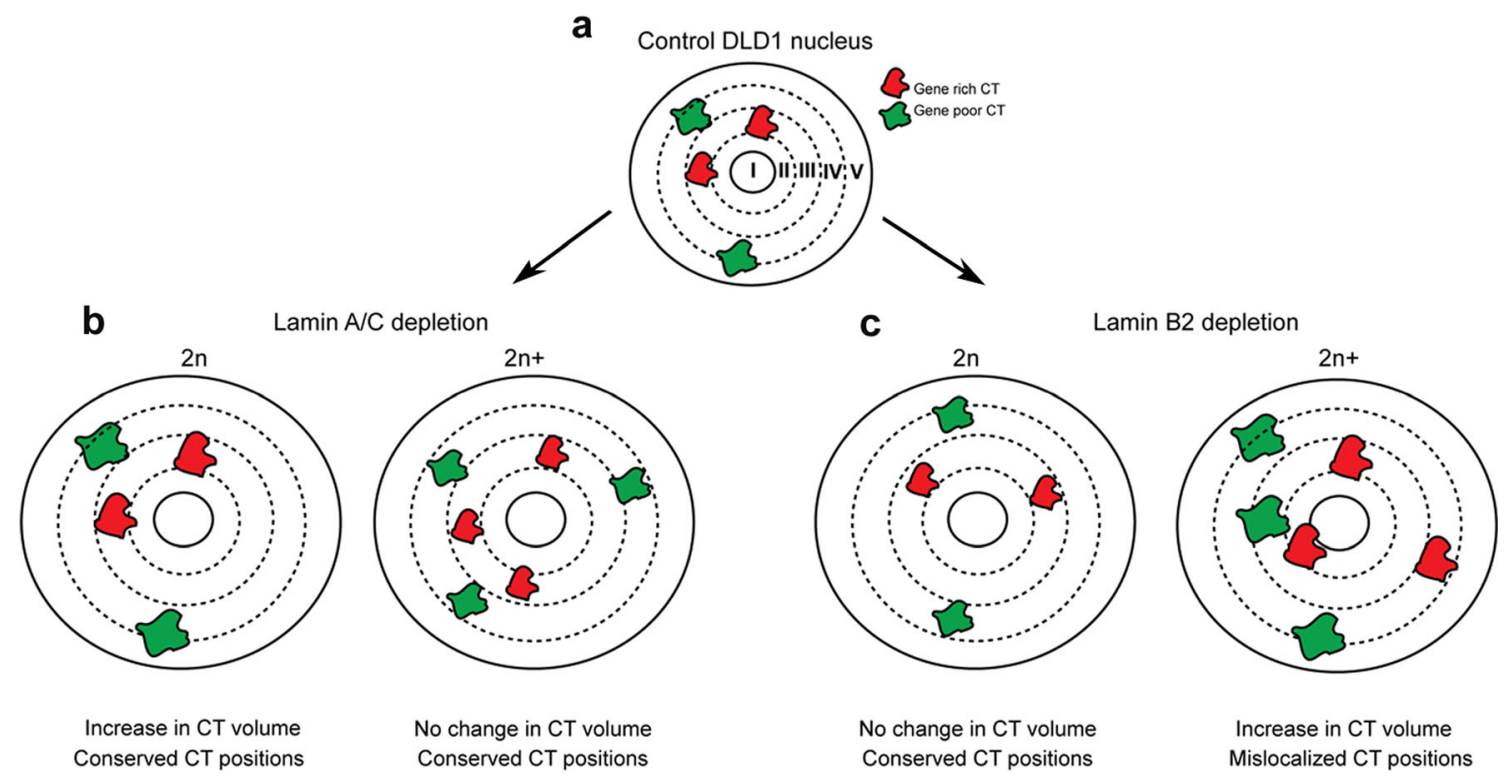

Fig. 8 Lamin B2 depletion perturbs positioning constraints of aneuploid chromosome territories in the interphase nucleus. a Schematic representation of the nucleus divided into five concentric sub-shells (IV) of $\sim 20 \%$ radial distance. Gene-rich CT occupies the nuclear interior (sub-shell: III), while gene-poor CT occupies the nuclear periphery (subshell: IV). Nuclear volume shows an increase in both Lamin A/C and Lamin B2 Kd. b Representation of the diploid (2n) and the aneuploid

$(2 \mathrm{n}+)$ nucleus upon Lamin A/C Kd. Both diploid and aneuploid CTs show conserved positions, while diploid CTs show a significant increase in volume upon Lamin A/C Kd. $\mathbf{c}$ Representation of the diploid ( $2 \mathrm{n}$ ) and the aneuploid $(2 \mathrm{n}+)$ nucleus upon Lamin B2 Kd. Diploid CTs show conserved chromosome positions and no change in volume, while aneuploid CTs show mislocalization of CTs and significant change in volume upon Lamin B2 Kd

However, our studies reveal that the volumes of aneuploid CTs are comparable irrespective of the nuclear space (subshells) that they occupy (Fig. 7). Notably, diploid CTs showed an increase in volume upon Lamin $\mathrm{A} / \mathrm{C} \mathrm{Kd}$ (Fig. 7). Gene expression profiling showed a downregulation of SMC1A ( 2-fold) upon Lamin A/C but not in Lamin B2 knockdown. SMC1A regulates chromatin architecture and could potentially impinge on volumes of CTs upon Lamin A/C depletion (Phillips-Cremins et al. 2013). Essentially, our data suggests that maintenance of $\mathrm{CT}$ position and function is not just dependent on gene density but additionally requires Lamins and its interactors to function as the "zip code" for the correct placement and regulation of transcriptional activity of either diploid or aneuploid CTs in the interphase nucleus.

The altered position of the aneuploid chromosomes could potentially have a bearing on their interaction with the nuclear lamina and with neighboring CTs (Branco and Pombo 2006). Haploid and diploid KBM7 cells show variability in their LAD profiles, suggesting that LAD profiles are modulated by the ploidy of cells (Kind et al. 2015). It would be useful to compare LAD profiles in diploid versus aneuploid Lamindepleted cells, although a potential technical limitation is to specifically enrich number of cells with chromosomal aneuploidies. In addition, the identification of chromatin contacts in single cells by Hi-C studies and RNA-Seq analyses would enable assessing the impact of chromosomal aneuploidies on chromatin contact frequencies and transcription

(Hashimshony et al. 2012; Islam et al. 2012; Nagano et al. 2013).

Whole chromosomal gains in colorectal cancer cell lines and patient samples positively correlate with gene expression levels (Grade et al. 2007). Gene expression profiling of human Chr.5 and Chr.7 in colorectal cancer cells (HCT116 and DLD1 cells, respectively), Chr.13 trisomy (Edward's syndrome), and Chr.21 trisomy in Down's syndrome showed an upregulation of transcripts from the aneuploid chromosomes by $\sim 1.1-1.5$ fold (FitzPatrick et al. 2002; Mao et al. 2003; Stingele et al. 2012; Upender et al. 2004). Lamin depletion followed by gene expression profiling of Lamin A/C and B2 Kd DLD1 cells showed an $\sim 1 \%$ deregulation of the transcriptome in a nonoverlapping manner (Fig. 2a). It remains to be examined if aneuploid chromosomes are transcriptionally active even when they are mislocalized in the interphase nucleus in Lamin-depleted cells.

Relatively fewer studies have previously examined the correlation between the spatial organization and expression status of gene loci in aneuploid cells. Amplified C-MYC (Chr.8q24) gene loci are localized external to chromosome 8 territory in colon cancer cell line HT-29 (Harnicarova et al. 2006). Interestingly, these loci were found to be transcriptionally active. The lamina is a transcriptionally repressive zone, and repositioning of gene loci away from the nuclear lamina is associated with an increase in its gene expression levels (Peric-Hupkes et al. 2010; Shachar et al. 2015), as shown for artificially targeted reporter genes ( $\mathrm{LacO}$ gene visualized 
using LacI-GFP) in single cells (Finlan et al. 2008; Reddy et al. 2008) as well as an endogenous gene TCRB, in contact with the nuclear Lamina (Schlimgen et al. 2008; Shachar et al. 2015). Our studies reveal altered spatial organization of gene loci consistent with an increase in its expression levels in Lamin B2-depleted cells. The candidate gene locus ZNF570 was not only repositioned away from the nuclear lamina in both diploid and aneuploid cells but also showed a significant increase in transcript signals in Lamin B2-depleted cells (Figs. 6 and S9).

Taken together, our studies suggest that chromosomal aneuploidies induced in Lamin-deficient cells may achieve enhanced transcriptional deregulation by sampling diverse subnuclear microenvironments in the interphase nucleus. Conversely, the presence of Lamins serves to not only prevent chromosomal aneuploidies but also dampen their spatial excursions in the nucleus. A thorough understanding of transcriptional outputs of aneuploid chromosomes in the context of their spatial organization would undoubtedly have farreaching consequences on the mechanisms of cancer initiation and diseases associated with chromosomal aneuploidies.

Acknowledgments We gratefully acknowledge facilities provided by IISER, Pune. We acknowledge members of the chromosome biology lab for their critical comments on the manuscript. Authors thank Genotypic, Bangalore, for gene expression profiling using microarrays and Bionivid, Bangalore, for microarray data analyses. We thank Wellcome Trust-DBT India Alliance for all their support. We sincerely thank all the referees for their critical inputs and suggestions on the manuscript.

\section{Compliance with ethical standards}

Funding This work was supported by funding from the Wellcome Trust-DBT India Alliance intermediate fellowship (Grant No. 30711044) awarded to K.S. and intramural funding from IISER Pune. D.R. acknowledges a fellowship from Council of Scientific and Industrial Research (CSIR, New Delhi).

Conflict of interest The authors declare that they have no conflict of interest.

Ethical approval This article does not contain any studies with human participants or animals performed by any of the authors.

Open Access This article is distributed under the terms of the Creative Commons Attribution 4.0 International License (http:// creativecommons.org/licenses/by/4.0/), which permits unrestricted use, distribution, and reproduction in any medium, provided you give appropriate credit to the original author(s) and the source, provide a link to the Creative Commons license, and indicate if changes were made.

\section{References}

Adam SA, Goldman RD (2012) Insights into the differences between the A- and B-type nuclear lamins. Adv Biol Regul 52:108-113. doi:10. 1016/j.advenzreg.2011.11.001
Bardi G, Sukhikh T, Pandis N, Fenger C, Kronborg O, Heim S (1995) Karyotypic characterization of colorectal adenocarcinomas. Genes Chromosomes Cancer 12:97-109

Belmont AS, Zhai Y, Thilenius A (1993) Lamin B distribution and association with peripheral chromatin revealed by optical sectioning and electron microscopy tomography. J Cell Biol 123:1671-1685

Belt EJ et al (2011) Loss of lamin A/C expression in stage II and III colon cancer is associated with disease recurrence. Eur J Cancer 47:18371845. doi:10.1016/j.ejca.2011.04.025

Bracken AP, Dietrich N, Pasini D, Hansen KH, Helin K (2006) Genomewide mapping of Polycomb target genes unravels their roles in cell fate transitions. Genes Dev 20:1123-1136. doi:10.1101/gad.381706

Branco MR, Pombo A (2006) Intermingling of chromosome territories in interphase suggests role in translocations and transcriptiondependent associations. PLoS Biol 4:e138. doi:10.1371/journal. pbio. 0040138

Burke B, Stewart CL (2002) Life at the edge: the nuclear envelope and human disease. Nat Rev Mol Cell Biol 3:575-585. doi:10.1038/ nrm879

Butin-Israeli V et al (2015) Role of lamin b1 in chromatin instability. Mol Cell Biol 35:884-898. doi:10.1128/MCB.01145-14

Camps J, Wangsa D, Falke M, Brown M, Case CM, Erdos MR, Ried T (2014) Loss of lamin B1 results in prolongation of S phase and decondensation of chromosome territories. FASEB J 28:34233434. doi:10.1096/fj.14-250456

Chambeyron S, Bickmore WA (2004) Chromatin decondensation and nuclear reorganization of the HoxB locus upon induction of transcription. Genes Dev 18:1119-1130. doi:10.1101/gad.292104

Chaumeil J, Augui S, Chow JC, Heard E (2008) Combined immunofluorescence, RNA fluorescent in situ hybridization, and DNA fluorescent in situ hybridization to study chromatin changes, transcriptional activity, nuclear organization, and X-chromosome inactivation. Methods Mol Biol 463:297-308. doi:10.1007/978-1-59745-406$3 \_18$

Cimini D, Degrassi F (2005) Aneuploidy: a matter of bad connections. Trends Cell Biol 15:442-451. doi:10.1016/j.tcb.2005.06.008

Clements L, Manilal S, Love DR, Morris GE (2000) Direct interaction between emerin and lamin A. Biochem Biophys Res Commun 267: 709-714. doi:10.1006/bbrc.1999.2023

Constantinescu D, Gray HL, Sammak PJ, Schatten GP, Csoka AB (2006) Lamin $\mathrm{A} / \mathrm{C}$ expression is a marker of mouse and human embryonic stem cell differentiation. Stem Cells 24:177-185. doi:10.1634/ stemcells.2004-0159

Cremer $\mathrm{M}$ et al (2001) Non-random radial higher-order chromatin arrangements in nuclei of diploid human cells. Chromosome Res 9: $541-567$

Cremer M et al (2003) Inheritance of gene density-related higher order chromatin arrangements in normal and tumor cell nuclei. J Cell Biol 162:809-820. doi:10.1083/jcb.200304096

Croft JA, Bridger JM, Boyle S, Perry P, Teague P, Bickmore WA (1999) Differences in the localization and morphology of chromosomes in the human nucleus. J Cell Biol 145:1119-1131

de Las Heras JI, Meinke P, Batrakou DG, Srsen V, Zuleger N, Kerr AR, Schirmer EC (2013) Tissue specificity in the nuclear envelope supports its functional complexity. Nucleus 4:460-477. doi:10.4161/ nucl.26872

Dechat T, Korbei B, Vaughan OA, Vlcek S, Hutchison CJ, Foisner R (2000) Lamina-associated polypeptide 2alpha binds intranuclear A-type lamins. J Cell Sci 113(Pt 19):3473-3484

Dechat T, Gesson K, Foisner R (2010) Lamina-independent lamins in the nuclear interior serve important functions. Cold Spring Harb Symp Quant Biol 75:533-543. doi:10.1101/sqb.2010.75.018

Dixon JR et al (2012) Topological domains in mammalian genomes identified by analysis of chromatin interactions. Nature 485:376-380. doi:10.1038/nature11082 
Finlan LE et al (2008) Recruitment to the nuclear periphery can alter expression of genes in human cells. PLoS Genet 4:e1000039. doi: 10.1371/journal.pgen.1000039

FitzPatrick DR, Ramsay J, McGill NI, Shade M, Carothers AD, Hastie ND (2002) Transcriptome analysis of human autosomal trisomy. Hum Mol Genet 11:3249-3256

Foisner R, Gerace L (1993) Integral membrane proteins of the nuclear envelope interact with lamins and chromosomes, and binding is modulated by mitotic phosphorylation. Cell 73:1267-1279

Foster HA, Abeydeera LR, Griffin DK, Bridger JM (2005) Non-random chromosome positioning in mammalian sperm nuclei, with migration of the sex chromosomes during late spermatogenesis. J Cell Sci 118:1811-1820. doi:10.1242/jcs.02301

Foster CR, Robson JL, Simon WJ, Twigg J, Cruikshank D, Wilson RG, Hutchison CJ (2011) The role of Lamin A in cytoskeleton organization in colorectal cancer cells: a proteomic investigation. Nucleus 2:434-443. doi:10.4161/nucl.2.5.17775, http://dx.doi.org/10. 4161/nucl.2.5.17775

Galiova G, Bartova E, Kozubek S (2004) Nuclear topography of beta-like globin gene cluster in IL-3-stimulated human leukemic K-562 cells. Blood Cells Mol Dis 33:4-14. doi:10.1016/j.bemd.2004.03.006

Gesson K, Rescheneder P, Skoruppa MP, von Haeseler A, Dechat T, Foisner R (2016) A-type lamins bind both hetero- and euchromatin, the latter being regulated by lamina-associated polypeptide 2 alpha. Genome Res. doi:10.1101/gr.196220.115

Goetze S et al (2007) The three-dimensional structure of human interphase chromosomes is related to the transcriptome map. Mol Cell Biol 27:4475-4487. doi:10.1128/MCB.00208-07

Goldman AE, Maul G, Steinert PM, Yang HY, Goldman RD (1986) Keratin-like proteins that coisolate with intermediate filaments of BHK-21 cells are nuclear lamins. Proc Natl Acad Sci U S A 83: 3839-3843

Goodman B, Channels W, Qiu M, Iglesias P, Yang G, Zheng Y (2010) Lamin B counteracts the kinesin Eg5 to restrain spindle pole separation during spindle assembly. J Biol Chem 285:35238-35244. doi: 10.1074/jbc.M110.140749

Grade $M$ et al (2007) Gene expression profiling reveals a massive, aneuploidy-dependent transcriptional deregulation and distinct differences between lymph node-negative and lymph node-positive colon carcinomas. Cancer Res 67:41-56. doi:10.1158/0008-5472. CAN-06-1514

Gruenbaum Y, Medalia O (2015) Lamins: the structure and protein complexes. Curr Opin Cell Biol 32:7-12. doi:10.1016/j.ceb.2014.09. 009

Guelen L et al (2008) Domain organization of human chromosomes revealed by mapping of nuclear lamina interactions. Nature 453 : 948-951. doi:10.1038/nature06947

Guo Y, Kim Y, Shimi T, Goldman RD, Zheng Y (2014) Concentrationdependent lamin assembly and its roles in the localization of other nuclear proteins. Mol Biol Cell 25:1287-1297. doi:10.1091/mbc. E13-11-0644

Harnicarova A, Kozubek S, Pachernik J, Krejci J, Bartova E (2006) Distinct nuclear arrangement of active and inactive c-myc genes in control and differentiated colon carcinoma cells. Exp Cell Res 312: 4019-4035. doi:10.1016/j.yexcr.2006.09.007

Harr JC, Luperchio TR, Wong X, Cohen E, Wheelan SJ, Reddy KL (2015) Directed targeting of chromatin to the nuclear lamina is mediated by chromatin state and A-type lamins. J Cell Biol 208:33-52. doi: $10.1083 /$ jcb. 201405110

Hashimshony T, Wagner F, Sher N, Yanai I (2012) CEL-Seq: single-cell RNA-Seq by multiplexed linear amplification. Cell Rep 2:666-673. doi:10.1016/j.celrep.2012.08.003

Hervé B et al (2016) Aneuploidy: the impact of chromosome imbalance on nuclear organization and overall genome expression. Clin Genet. doi:10.1111/cge. 12731
Hoger TH, Zatloukal K, Waizenegger I, Krohne G (1990) Characterization of a second highly conserved B-type lamin present in cells previously thought to contain only a single B-type lamin. Chromosoma 100:67-69

Islam S, Kjallquist U, Moliner A, Zajac P, Fan JB, Lonnerberg P, Linnarsson S (2012) Highly multiplexed and strand-specific single-cell RNA 5' end sequencing. Nat Protoc 7:813-828. doi:10. 1038/nprot.2012.022

Kalhor R, Tjong H, Jayathilaka N, Alber F, Chen L (2012) Genome architectures revealed by tethered chromosome conformation capture and population-based modeling. Nat Biotechnol 30:90-98. doi: 10.1038/nbt.2057

Kind J et al (2013) Single-cell dynamics of genome-nuclear lamina interactions. Cell 153:178-192. doi:10.1016/j.cell.2013.02.028

Kind J et al (2015) Genome-wide maps of nuclear lamina interactions in single human cells. Cell 163:134-47. doi:10.1016/j.cell.2015.08. 040

Kochin V et al (2014) Interphase phosphorylation of lamin A. J Cell Sci 127:2683-2696. doi: $10.1242 /$ jcs. 141820

Kolb T, Maass K, Hergt M, Aebi U, Herrmann H (2011) Lamin A and lamin $\mathrm{C}$ form homodimers and coexist in higher complex forms both in the nucleoplasmic fraction and in the lamina of cultured human cells. Nucleus 2:425-433. doi:10.4161/nucl.2.5.17765

Kuga T et al (2014) Lamin B2 prevents chromosome instability by ensuring proper mitotic chromosome segregation. Oncog 3:e94. doi: 10.1038/oncsis. 2014.6

Lee KK, Haraguchi T, Lee RS, Koujin T, Hiraoka Y, Wilson KL (2001) Distinct functional domains in emerin bind lamin A and DNAbridging protein BAF. J Cell Sci 114:4567-4573

Lieberman-Aiden E et al (2009) Comprehensive mapping of long-range interactions reveals folding principles of the human genome. Science 326:289-293. doi:10.1126/science.1181369

Malhas A, Lee CF, Sanders R, Saunders NJ, Vaux DJ (2007) Defects in lamin B1 expression or processing affect interphase chromosome position and gene expression. J Cell Biol 176:593-603. doi:10. 1083/jcb.200607054

Mao R, Zielke CL, Zielke HR, Pevsner J (2003) Global up-regulation of chromosome 21 gene expression in the developing Down syndrome brain. Genomics 81:457-467

Martin C, Chen S, Jackson DA (2010) Inheriting nuclear organization: can nuclear lamins impart spatial memory during post-mitotic nuclear assembly? Chromosome Res 18:525-541. doi:10.1007/ s10577-010-9137-8

Meaburn KJ et al (2007) Primary laminopathy fibroblasts display altered genome organization and apoptosis. Aging Cell 6:139-153. doi:10. $1111 / j .1474-9726.2007 .00270 . x$

Mehta IS, Figgitt M, Clements CS, Kill IR, Bridger JM (2007) Alterations to nuclear architecture and genome behavior in senescent cells. Ann NY Acad Sci 1100:250-263. doi:10.1196/annals. 1395.027

Mehta IS, Kulashreshtha M, Chakraborty S, Kolthur-Seetharam U, Rao BJ (2013) Chromosome territories reposition during DNA damagerepair response. Genome Biol 14:R135. doi:10.1186/gb-2013-1412-r135

Meuleman W et al (2013) Constitutive nuclear lamina-genome interactions are highly conserved and associated with A/T-rich sequence. Genome Res 23:270-280. doi:10.1101/gr.141028.112

Mewborn SK et al (2010) Altered chromosomal positioning, compaction, and gene expression with a lamin $\mathrm{A} / \mathrm{C}$ gene mutation. PLoS One 5: e14342. doi:10.1371/journal.pone.0014342

Moir RD, Montag-Lowy M, Goldman RD (1994) Dynamic properties of nuclear lamins: lamin B is associated with sites of DNA replication. J Cell Biol 125:1201-1212

Moir RD, Yoon M, Khuon S, Goldman RD (2000) Nuclear lamins A and $\mathrm{B} 1$ : different pathways of assembly during nuclear envelope formation in living cells. J Cell Biol 151:1155-1168 
Moss SF et al (1999) Decreased and aberrant nuclear lamin expression in gastrointestinal tract neoplasms. Gut 45:723-729

Nagano T et al (2013) Single-cell Hi-C reveals cell-to-cell variability in chromosome structure. Nature 502:59-64. doi:10.1038/ nature 12593

Neusser M, Schubel V, Koch A, Cremer T, Muller S (2007) Evolutionarily conserved, cell type and species-specific higher order chromatin arrangements in interphase nuclei of primates. Chromosoma 116:307-320. doi:10.1007/s00412-007-0099-3

Nora EP et al (2012) Spatial partitioning of the regulatory landscape of the X-inactivation centre. Nature 485:381-385. doi:10.1038/ nature 11049

Ondrej V, Lukasova E, Krejci J, Matula P, Kozubek S (2008) Lamin A/C and polymeric actin in genome organization. Mol Cells 26:356-361

Paz N, Zabala A, Royo F, Garcia-Orad A, Zugaza JL, Parada LA (2013) Combined fluorescent-chromogenic in situ hybridization for identification and laser microdissection of interphase chromosomes. PLoS One 8:e60238. doi:10.1371/journal.pone.0060238

Peric-Hupkes D, van Steensel B (2010) Role of the nuclear lamina in genome organization and gene expression. Cold Spring Harb Symp Quant Biol 75:517-524. doi:10.1101/sqb.2010.75.014

Peric-Hupkes D et al (2010) Molecular maps of the reorganization of genome-nuclear lamina interactions during differentiation. Mol Cell 38:603-613. doi:10.1016/j.molcel.2010.03.016

Petrova NV, Yakutenko II, Alexeevski AV, Verbovoy VA, Razin SV, Iarovaia OV (2007) Changes in chromosome positioning may contribute to the development of diseases related to X-chromosome aneuploidy. J Cell Physiol 213:278-283. doi:10.1002/jcp.21118

Phillips-Cremins JE et al (2013) Architectural protein subclasses shape 3D organization of genomes during lineage commitment. Cell 153: 1281-1295. doi:10.1016/j.cell.2013.04.053

Qi R et al (2015) The lamin-A/C-LAP2alpha-BAF1 protein complex regulates mitotic spindle assembly and positioning. J Cell Sci 128: 2830-2841. doi:10.1242/jcs.164566

Reddy KL, Zullo JM, Bertolino E, Singh H (2008) Transcriptional repression mediated by repositioning of genes to the nuclear lamina. Nature 452:243-247. doi:10.1038/nature06727

Ried T et al (1996) Comparative genomic hybridization reveals a specific pattern of chromosomal gains and losses during the genesis of colorectal tumors. Genes Chromosomes Cancer 15:234-245. doi:10.1002/ (SICI) 1098-2264(199604)15:4<234::AID-GCC5>3.0.CO;2-2

Rober RA, Weber K, Osborn M (1989) Differential timing of nuclear lamin $\mathrm{A} / \mathrm{C}$ expression in the various organs of the mouse embryo and the young animal: a developmental study. Development 105: 365-378

Roth U et al (2010) Differential expression proteomics of human colorectal cancer based on a syngeneic cellular model for the progression of adenoma to carcinoma. Proteomics 10:194-202. doi:10.1002/ pmic. 200900614

Schlimgen RJ, Reddy KL, Singh H, Krangel MS (2008) Initiation of allelic exclusion by stochastic interaction of Tcrb alleles with repressive nuclear compartments. Nat Immunol 9:802-809. doi:10.1038/ ni. 1624

Sengupta K et al (2007) Artificially introduced aneuploid chromosomes assume a conserved position in colon cancer cells. PLoS One 2: e199. doi:10.1371/journal.pone.0000199

Shachar S, Voss TC, Pegoraro G, Sciascia N, Misteli T (2015) Identification of gene positioning factors using high-throughput imaging mapping. Cell 162:911-923. doi:10.1016/j.cell.2015.07.035

Shete A, Rao P, Pati D, Merchant F (2014) Spatial quantitation of FISH signals in diploid versus aneuploid nuclei. Cytometry A 85:339352. doi:10.1002/cyto.a.22426

Shimi T et al (2008) The A- and B-type nuclear lamin networks: microdomains involved in chromatin organization and transcription. Genes Dev 22:3409-3421. doi:10.1101/gad.1735208
Shimi T et al (2011) The role of nuclear lamin B1 in cell proliferation and senescence. Genes Dev 25:2579-2593. doi:10.1101/gad.179515. 111

Shimi T et al (2015) Structural organization of nuclear Lamins A, C, B1 and $\mathrm{B} 2$ revealed by super-resolution microscopy. Mol Biol Cell. doi: 10.1091/mbc.E15-07-0461

Shumaker DK et al (2008) The highly conserved nuclear lamin Ig-fold binds to PCNA: its role in DNA replication. J Cell Biol 181:269 280. doi:10.1083/jcb.200708155

Smith S, Blobel G (1994) Colocalization of vertebrate lamin B and lamin $B$ receptor (LBR) in nuclear envelopes and in LBR-induced membrane stacks of the yeast Saccharomyces cerevisiae. Proc Natl Acad Sci U S A 91:10124-10128

Solovei I et al (2009) Nuclear architecture of rod photoreceptor cells adapts to vision in mammalian evolution. Cell 137:356-368. doi: 10.1016/j.cell.2009.01.052

Solovei I et al (2013) LBR and lamin A/C sequentially tether peripheral heterochromatin and inversely regulate differentiation. Cell 152: 584-598. doi:10.1016/j.cell.2013.01.009

Spann TP, Goldman AE, Wang C, Huang S, Goldman RD (2002) Alteration of nuclear lamin organization inhibits RNA polymerase II-dependent transcription. J Cell Biol 156:603-608. doi:10.1083/ jcb. 200112047

Stingele S, Stoehr G, Peplowska K, Cox J, Mann M, Storchova Z (2012) Global analysis of genome, transcriptome and proteome reveals the response to aneuploidy in human cells. Mol Syst Biol 8:608. doi:10. $1038 / \mathrm{msb} .2012 .40$

Swift J et al (2013) Nuclear lamin-A scales with tissue stiffness and enhances matrix-directed differentiation. Science 341:1240104. doi:10.1126/science. 1240104

Szczerbal I, Foster HA, Bridger JM (2009) The spatial repositioning of adipogenesis genes is correlated with their expression status in a porcine mesenchymal stem cell adipogenesis model system. Chromosoma 118:647-663. doi:10.1007/s00412-009-0225-5

Tagawa Y, Sawai T, Nakagoe T, Morinaga M, Yasutake T, Ayabe H, Tomita M (1996) Numerical aberrations of chromosomes 11 and 17 in colorectal adenocarcinomas. Surg Today 26:869-874

Taimen P et al (2009) A progeria mutation reveals functions for lamin A in nuclear assembly, architecture, and chromosome organization. Proc Natl Acad Sci U S A 106:20788-20793. doi:10.1073/pnas. 0911895106

Tanabe $\mathrm{H}$ et al (2002) Evolutionary conservation of chromosome territory arrangements in cell nuclei from higher primates. Proc Natl Acad Sci U S A 99:4424-4429. doi:10.1073/pnas.072618599

Tang CW et al (2008) The integrity of a lamin-B1-dependent nucleoskeleton is a fundamental determinant of RNA synthesis in human cells. J Cell Sci 121:1014-1024. doi:10.1242/jcs.020982

Towbin BD et al (2012) Step-wise methylation of histone H3K9 positions heterochromatin at the nuclear periphery. Cell 150:934-947. doi:10. 1016/j.cell.2012.06.051

Tsai MY, Wang S, Heidinger JM, Shumaker DK, Adam SA, Goldman RD, Zheng Y (2006) A mitotic lamin B matrix induced by RanGTP required for spindle assembly. Science 311:1887-1893. doi:10. $1126 /$ science. 1122771

Upender MB, Habermann JK, McShane LM, Korn EL, Barrett JC, Difilippantonio MJ, Ried T (2004) Chromosome transfer induced aneuploidy results in complex dysregulation of the cellular transcriptome in immortalized and cancer cells. Cancer Res 64:69416949. doi:10.1158/0008-5472.CAN-04-0474

van Berkum NL et al (2010) Hi-C: a method to study the threedimensional architecture of genomes. J Vis Exp. doi:10.3791/1869

Volpi EV et al (2000) Large-scale chromatin organization of the major histocompatibility complex and other regions of human chromosome 6 and its response to interferon in interphase nuclei. J Cell Sci 113(Pt 9):1565-1576 
Williamson I et al (2014) Spatial genome organization: contrasting views from chromosome conformation capture and fluorescence in situ hybridization. Genes Dev 28:2778-2791. doi:10.1101/gad.251694. 114

Willis ND et al (2008) Lamin A/C is a risk biomarker in colorectal cancer. PLoS One 3:e2988. doi:10.1371/journal.pone.0002988

Wu Z, Wu L, Weng D, Xu D, Geng J, Zhao F (2009) Reduced expression of lamin $\mathrm{A} / \mathrm{C}$ correlates with poor histological differentiation and prognosis in primary gastric carcinoma. J Exp Clin Cancer Res 28: 8. doi:10.1186/1756-9966-28-8

Yang SH, Jung HJ, Coffinier C, Fong LG, Young SG (2011) Are B-type lamins essential in all mammalian cells? Nucleus 2:562-569. doi:10. 4161/nucl.2.6.18085
Ye Q, Callebaut I, Pezhman A, Courvalin JC, Worman HJ (1997) Domain-specific interactions of human HP1-type chromodomain proteins and inner nuclear membrane protein LBR. J Biol Chem 272:14983-14989

Yusufzai TM, Tagami H, Nakatani Y, Felsenfeld G (2004) CTCF tethers an insulator to subnuclear sites, suggesting shared insulator mechanisms across species. Mol Cell 13:291-298

Zewe M, Hoger TH, Fink T, Lichter P, Krohne G, Franke WW (1991) Gene structure and chromosomal localization of the murine lamin B2 gene. Eur J Cell Biol 56:342-350

Zullo JM et al (2012) DNA sequence-dependent compartmentalization and silencing of chromatin at the nuclear lamina. Cell 149:14741487. doi:10.1016/j.cell.2012.04.035 